\title{
DUALITY FOR DIFFERENTIAL-DIFFERENCE SYSTEMS OVER LIE GROUPS
}

\author{
HENRI BOURLÈ** AND ULRICH OBERST ${ }^{\dagger}$
}

\begin{abstract}
In modern mathematical systems theory, there exist two consistent ways of defining and describing a linear system: (i) in the behavioral approach, a linear system is the kernel $\mathfrak{B}$ of a matrix-valued operator $R$ in a power of a signal space $W$; (ii) in the module-theoretic setting, a linear system is the cokernel $M$ of the above matrix $R$. These two formulations have connections. The minimal conditions under which they are equivalent are investigated in this paper. The general theory is applied to differential-difference systems over Lie groups.
\end{abstract}

Key words. module, behavior, duality, controllability, differential-difference system

AMS subject classification. 93B25.

1. Introduction. The notion of linear system can be defined in many ways (by a transfer function, a state-space representation, etc.). Two of them are of a particular interest since they are complete (especially, hidden modes are well represented) and intrinsic (i.e. independent of a choice made a priori, e.g. of the state and of the inputand output-variables). They can be characterized as follows, denoting by $\mathbf{A}$ a ring of operators:

(i) A linear system is characterized by its behavior, i.e. the kernel $\mathfrak{B}$ of a matrixvalued operator $R \in \mathbf{A}^{q \times k}$ in a power of a solution space $W$, assumed to be an A-module. This approach was initiated by Willems in the 80's [68] (see also [70]).

(ii) A linear system is associated with a finitely presented $\mathbf{A}$-module $M$, i.e. the cokernel of a matrix-valued operator $R$ such as the one above. This formulation was initiated by Malgrange [46] and developed by experts in the theory of $\mathcal{D}$-modules (e.g., Palamodov, Björk, Kashiwara and Schapira, to cite a few of them: see [43], [21], [3], [55] and the references therein) and was popularized in systems theory by Fliess $[29]$.

Each of these two above approaches has its advantages. Approach (i) emphasizes the role of solutions and therefore could be more appealing for engineers. Approach (ii) emphasizes the role of equations which are the base of science. Given the ring $\mathbf{A}$, the behavior $\mathfrak{B}$ can always be deduced from the module $M$ and the signal space $W$, thus it can be written $\mathfrak{B}_{W}(M)$; for the converse to hold true, the space $W$ must be "rich enough" (for example, if $W=\{0\}$, then $\mathfrak{B}_{W}(M)=\{0\}$ whatever the module $M$ is). On the other hand, the ring $\mathbf{A}$ must be appropriate both to reflect the nature of the system and for the equations over $\mathbf{A}$ to have solutions in the suitable space $W$, thus there must be the right interplay between $\mathbf{A}$ and $W$. If $W$ is a cogenerator -a classical notion in homological algebra-, then $M$ is determined by $\mathfrak{B}_{W}(M)$ since the map $M \mapsto \mathfrak{B}_{W}(M)$ is injective. The second author extensively used the notion of cogenerator and showed its importance in systems theory (see [50] and related references).

However, the condition that $W$ be a cogenerator is somewhat restrictive (as already noticed by Wood [72]). The aim of this paper is to further investigate the minimal conditions under which approaches (i) and (ii) are equivalent. This leads us

*SATIE, ENS Cachan/CNAM, 61 Avenue President Wilson, F-94230 Cachan, France (e-mail: Henri.Bourles@satie.ens-cachan.fr; tel: +331409588 83).

${ }^{\dagger}$ Institute für Mathematik, Universität Innsbruck, Technikerstrasse 13, A-6020 Innsbruck, Austria (e-mail: Ulrich.Oberst@uibk.ac.at). 
to enlarge the notion of cogenerator, usually defined in (and for) an abelian category $\mathcal{C}$ (such as ${ }_{\mathbf{A}} \mathbf{M o d}$, the category of left $\mathbf{A}$-modules). Here we define a cogenerator in an additive category $\mathcal{C}$ for a full subcategory $\mathcal{D}$ of $\mathcal{C}$. A typical example is when $\mathcal{C}$ is ${ }_{\mathbf{A}} \mathbf{M o d}$ and $\mathcal{D}$ is ${ }_{\mathbf{A}} \mathbf{M o d}^{f p}$, the category of finitely presented left $\mathbf{A}$-modules.

Then we apply the general theory to differential-difference systems over Lie groups. Such systems -and, more generally, convolution systems over Lie groupshave applications in various fields such as robotics, image processing, vision and polymer science (see, e.g., [14], [75] and [16]).

The paper is organized as follows: the general definition of a cogenerator for a subcategory is given in Section 2 after some preliminaries, and illustrated by two examples: that already mentioned $\left(\mathcal{C}={ }_{\mathbf{A}} \mathbf{M o d}, \mathcal{D}={ }_{\mathbf{A}} \mathbf{M o d}^{f p}\right)$ and another one, similar but with additional topological considerations. The application to differential systems with shifts over a Lie group is developed in Sections 3 (case of lumped shifts) and 4 (case of distributed shifts). Section 5 is devoted to concluding remarks. The main results are stated in theorems, the other ones in lemmas and corollaries. Two appendices are proposed in Section 6 for the reader's convenience: the first one on categories and functors, the second one on Lie groups, Lie algebras and convolution algebras over Lie groups.

ACKNOWLEDGEMENT 1.1. The authors would like to thank the anonymous reviewers, as well as Prof. M. Duflo, N. Ortner, F. Rouvière, and P. Wagner, whose comments were helpful to improve the manuscript.

\section{Cogenerators: a categorical point of view.}

\subsection{A general definition of a cogenerator.}

2.1.1. $U$-cokernels and behaviors. The following notation is used throughout this paper:

Notation 2.1. Let $\mathbf{R}$ be a ring. (i) The morphisms of left (resp. right) $\mathbf{R}$ modules are left (resp. right) morphisms (see Appendix 1). (ii) Let $M$ be a left (resp. right) $\mathbf{R}$-module and consider a power and a copower of $M$ with set of indices $I$; the power is denoted by $M^{I}$ (resp. $\left.{ }^{I} M\right)$, the copower by $M^{(I)}$ (resp. $\left.{ }^{(I)} M\right)$, and both of them by $M^{n}$ (resp. ${ }^{n} M$ ) if $I=\{1, \ldots, n\}$; the elements of this power and of this copower are represented by rows (resp. columns) and the set of all $n \times m$ matrices with entries in $M$ is denoted by ${ }^{n} M^{m}$. (iii) The set of nonzero elements of $\mathbf{R}$ is denoted by $\mathbf{R}^{\times}$.

In what follows, $\mathcal{C}$ is a preabelian category with arbitrary products (see Appendix 1), except when otherwise stated. Let $U$ and $W$ be objects of $\mathcal{C}, \mathbf{A} \triangleq \operatorname{Hom}_{\mathcal{C}}(U, U)$ and $\mathbf{E} \triangleq \operatorname{Hom}_{\mathcal{C}}(W, W)$ the rings of left endomorphisms of $U$ and $W$, respectively (Appendix $1, \S 6.1 .1)$. Then for any objects $M, N$ of $\mathcal{C}$, the abelian groups $\operatorname{Hom}_{\mathcal{C}}(M, W)$ and $\operatorname{Hom}_{\mathcal{C}}(U, N)$ of all left morphisms $\bullet f: M \rightarrow W$ and $\bullet g: U \rightarrow N$ are respectively a right $\mathbf{E}$-module and a left $\mathbf{A}$-module via composition of morphisms. The abelian group $\operatorname{Hom}_{\mathcal{C}}(U, W)$ is an (A, E)-bimodule ([4], Subsect. II.1.14). The additive functor $\mathfrak{B}_{W}=\operatorname{Hom}_{\mathcal{C}}(\bullet, W)$ is contravariant and left exact from $\mathcal{C}$ to the category $\operatorname{Mod}_{\mathbf{E}}$ of right $\mathbf{E}$-modules.

Let $I$ be a set of indices, arbitrary if $\mathcal{C}$ has arbitrary coproducts and finite otherwise. Consider a subobject $N$ of $U^{(I)}$ (written $N \subseteq U^{(I)}$ ), $: N \hookrightarrow U^{(I)}$ the inclusion, and the associated factor object $M=\operatorname{coker} \iota$ (also written $M=U^{(I)} / N$ ) defined by the canonical exact sequence of left morphisms

$$
N \stackrel{\bullet}{\longrightarrow} U^{(I)} \stackrel{\bullet \varphi}{\longrightarrow} M \longrightarrow 0 .
$$


Definition 2.2. (i) A factor of $U^{(I)}$ is called $U$-generated (and $U$-finitely generated if $I$ is finite).

(ii) The above object $M=$ coker $\iota$ is called a $U$-cokernel.

REMARK 2.3. (i) All objects of $\mathcal{C}$ are $U$-generated if, and only if $U$ is a generator. If the category $\mathcal{C}$ is not abelian, a $U$-generated object need not be a $U$-cokernel since Noether's first isomorphism theorem is not valid (see also Remark 2.22 below).

(ii) The above item holds because we allow the set I of indices to be arbitrary. This is also necessary for the proof of Theorem 2.13(iii) and thus for that of Theorem 4.5(iv) below to be correct.

As in Appendix 1, $\$ 6.1 .2$, the right E-modules ${ }^{I} \operatorname{Hom}_{\mathcal{C}}(U, W)$ and $\operatorname{Hom}_{\mathcal{C}}\left(U^{(I)}, W\right)$ are identified. The subobject $N$ gives rise to the $W$-behavior

$$
N^{\perp} \triangleq\left\{w \in \operatorname{Hom}_{\mathcal{C}}\left(U^{(I)}, W\right): \iota w=0\right\}
$$

which is an object of $\operatorname{Mod}_{\mathbf{E}}$. The result below is a straightforward generalization of a classical result due to Malgrange ([46], Sect. 2, $\left.2^{\circ}\right)$ where multidimensional systems with constant coefficients are considered:

Lemma 2.4. There is a canonical $\mathbf{E}$-linear isomorphism

$$
\sigma: \operatorname{Hom}_{\mathcal{C}}(M, W) \stackrel{\sim}{\rightarrow} N^{\perp}: \hat{w} \mapsto w
$$

given by $w=\varphi \hat{w}$.

Proof. The kernel ker $\mathfrak{B}_{W}(\iota)$ is the largest subobject of $\mathfrak{B}_{W}\left(U^{(I)}\right)=$ ${ }^{I} \operatorname{Hom}_{\mathcal{C}}(U, W)$ annihilated by $\mathfrak{B}_{W}(\iota): w \mapsto \iota w$ (Appendix 1, $\S 6.1 .1$ ), thus $N^{\perp}$ is a representative of $\operatorname{ker} \mathfrak{B}_{W}(\iota)$. Applying $\mathfrak{B}_{W}$ to $(2.1)$ we obtain the exact sequence of right morphisms

$$
0 \longrightarrow \mathfrak{B}_{W}(M) \stackrel{\mathfrak{B}_{W}(\varphi)}{\longrightarrow} \mathfrak{B}_{W}\left(U^{(I)}\right) \stackrel{\mathfrak{B}_{W}(\iota)}{\longrightarrow} \mathfrak{B}_{W}(N)
$$

thus $\mathfrak{B}_{W}(M)$ is another representative of $\operatorname{ker} \mathfrak{B}_{W}(\iota)$. The isomorphism $\sigma: \hat{w} \mapsto \varphi \hat{w}$ is canonical and $\mathbf{E}$-linear.

Definition 2.5. Let $\mathcal{D}$ be a full subcategory of $\mathcal{C}$, all objects of which are $U$ cokernels. The class of all $W$-behaviors associated with $\mathcal{D}$ is $\mathfrak{B}_{W}(\mathcal{D})$.

Conversely, let $\mathfrak{B} \subseteq \operatorname{Hom}_{\mathcal{C}}\left(U^{(I)}, W\right)$ and consider the object

$$
\mathfrak{B}^{\perp} \triangleq \bigcap_{w \in \mathfrak{B}} \operatorname{ker} w=\operatorname{ker}\left((w)_{w \in \mathfrak{B}}: U^{(I)} \longrightarrow W^{\mathfrak{B}}\right) \subseteq U^{(I)} .
$$

For any $U$-cokernel $M$ (i.e. for any $M$ such as in (2.1)) and $\mathfrak{B}=N^{\perp}$ defined by (2.2) we define the canonical (left) morphism

$$
\text { • } \phi_{M} \triangleq(\hat{w})_{w \in \mathfrak{B}}: M \longrightarrow W^{\mathfrak{B}}
$$

we have by Lemma $2.4 \varphi \phi_{M}=(w)_{w \in \mathfrak{B}}$, thus by (2.4)

$$
\operatorname{ker}\left(\bullet \varphi \phi_{M}\right)=\mathfrak{B}^{\perp} .
$$

Lemma 2.6. The two maps $(\bullet)^{\perp}: N \mapsto N^{\perp}$ and $\mathfrak{B} \mapsto \mathfrak{B}^{\perp}$ constitute an orderreversing Galois connection (for the inclusion). 
Proof. (i) Let $N_{1} \subseteq N_{2}$ and $\iota_{1}: N_{1} \hookrightarrow U^{(I)}$ the inclusion. For any $w \in N_{2}^{\perp}$, $\iota_{1} w=0$, thus $N_{2}^{\perp} \subseteq N_{1}^{\perp}$. Similarly, $\mathfrak{B}_{2}^{\perp} \subseteq \mathfrak{B}_{1}^{\perp}$ whenever $\mathfrak{B}_{1} \subseteq \mathfrak{B}_{2}$. (ii) We have to prove that

$$
N \subseteq N^{\perp \perp}, \quad \mathfrak{B} \subseteq \mathfrak{B}^{\perp \perp}
$$

We have $N \subseteq \operatorname{ker} \varphi \subseteq \operatorname{ker} \varphi \phi_{M}=N^{\perp \perp}$ and the proof of the second inclusion is similar.. $\square$

2.1.2. Cogenerators. First let us establish the following:

LEMMA 2.7. Let $\mathcal{C}$ be a preabelian (or more generally an additive) category with arbitrary products, $M$ and $W$ objects of $\mathcal{C}$, and the canonical morphism

$$
\bullet \phi_{M} \triangleq(\hat{w})_{\hat{w} \in \operatorname{Hom}_{\mathcal{C}}(M, W)}: M \rightarrow W^{\operatorname{Hom}_{\mathcal{C}}(M, W)} .
$$

(1) The following conditions are equivalent:

(i) $\phi_{M}$ is a monomorphism;

(ii) There exists a monomorphism $M \hookrightarrow W^{J}$ for some set of indices $J$;

(iii) For every object $M_{1}$ of $\mathcal{C}$, the arrow map

$$
\mathfrak{B}_{W}: \operatorname{Hom}_{\mathcal{C}}\left(M_{1}, M\right) \mapsto \operatorname{Hom}_{\mathbf{E}}\left(\mathfrak{B}_{W}(M), \mathfrak{B}_{W}\left(M_{1}\right)\right)
$$

is injective.

(2) If $U$ is a generator, the above conditions are equivalent to:

(iv) For any nonzero morphism $\bullet h: U \rightarrow M$, there exists $\bullet \beta: M \rightarrow W$ with $\bullet h \beta \neq 0$.

Proof. (1). (i) $\Rightarrow\left(\right.$ ii): This is clear (take $J=\operatorname{Hom}_{\mathcal{C}}(M, W)$ ). (ii) $\Rightarrow$ (iii): Let $\bullet f: M_{1} \rightarrow M$ and $\bullet \eta=\left(\bullet \eta_{j}\right)_{i \in J}: M \hookrightarrow W^{J}$. If $\mathfrak{B}_{W}(f)=0$, then $\mathfrak{B}_{W}(f)\left(\eta_{j}\right)=$ $\bullet f \eta_{j}=0$ for all $j \in J$, thus $\bullet f \eta=0$ and since $\bullet \eta$ is a monomorphism, this implies $f=0$. (iii) $\Rightarrow(\mathrm{i})$ : By $(2.5), \mathfrak{B}_{W}(f)=0$ if, and only if $f \phi_{M}=0$; thus, if $\mathfrak{B}_{W}$ is injective, then $\phi_{M}$ is a monomorphism.

(2). Assume that $U$ is a generator. (ii) $\Rightarrow\left(\right.$ iv): Let $\bullet \eta=\left(\bullet \eta_{j}\right)_{j \in J}: M \hookrightarrow W^{J}$ and $\bullet h: U \rightarrow M, h \neq 0$. Then $\bullet h \eta 0$, thus there exists $j \in J$ such that $h \eta_{j} \neq 0$ and we have found $\beta=\eta_{j}: M \rightarrow W$ such that $\bullet h \neq 0$. (iv) $\Rightarrow\left(\right.$ iii): Let $\bullet f: M_{1} \rightarrow M$ be such that $\mathfrak{B}_{W}(f)=0$, thus $\mathfrak{B}_{W}(f)(\beta)=f \beta=0$ for any $\bullet \beta: M \rightarrow W$. If $f \neq 0$, there exists $\bullet h_{1}: U \rightarrow M_{1}$ such that $h_{1} f \neq 0$ whereas $h_{1} f \beta=0$, i.e. $h \beta=0$ with $h=h_{1} f \neq 0$, and (iv) does not hold.

COROllary AND Definition 2.8. (i) Let $\mathcal{C}$ be a preabelian (or more generally an additive) category with arbitrary products. An object $W \in \mathcal{C}$ is called a cogenerator for a full subcategory $\mathcal{D}$ of $\mathcal{C}$ if the conditions of Lemma 2.7(1) are satisfied for all objects $M \in \mathcal{D}$.

(ii) Then $\mathfrak{B}_{W}(\mathcal{D})$ is a subcategory of $\operatorname{Mod}_{\mathbf{E}}$ and the functor $\mathfrak{B}_{W}$ induces an isomorphism $\mathfrak{B}_{W}: \mathcal{D}^{o p} \stackrel{\sim}{\rightarrow} \mathfrak{B}_{W}(\mathcal{D})$.

See Appendix 1 for the proof of (ii). When taking $\mathcal{D}=\mathcal{C}$, one meets the classical definition of a cogenerator again, and the statement of Lemma 2.7 can be easily generalized to the case when $\mathcal{C}$ is any category with arbitrary products (this generalization is left to the reader).

Recall that a left $\mathbf{A}$-module $M$ is said to be faithful if for any $0 \neq a \in \mathbf{A}, a M \neq 0$. We have the following:

LEMMA 2.9. Let $\mathcal{D}$ be a full subcategory of the preabelian (or more generally the additive) category $\mathcal{C}, W \in \mathcal{C}$ a cogenerator for $\mathcal{D}, U \in \mathcal{D}$ and $\mathbf{A} \triangleq \operatorname{Hom}_{\mathcal{C}}(U, U)$. Then $\mathfrak{B}_{W}(U)$ is a faithful left $\mathbf{A}$-module. 
Proof. By Lemma 2.7(1)(iii), the map

$$
\mathfrak{B}_{W}: \mathbf{A} \rightarrow \operatorname{Hom}_{\mathbf{E}}\left(\mathfrak{B}_{W}(U), \mathfrak{B}_{W}(U)\right), \quad a \mapsto a \bullet
$$

(where $a \bullet$ is the left composition by $a$ ) is injective, thus if $a$ is nonzero, then $a \bullet$ and $a \mathfrak{B}_{W}(U)$ are nonzero too, which implies that the left $\mathbf{A}$-module $\mathfrak{B}_{W}(U)$ is a faithful. 口

THEOREM 2.10. Let $\mathcal{C}$ be a semi-abelian category (Appendix 1, §6.1.1) and $U, W \in \mathcal{C}$.

(1) Let $I$ be a set of indices, $N \subseteq U^{(I)}, \iota: N \hookrightarrow U^{(I)}$ the inclusion and $M=U^{(I)} / N$ a $U$-cokernel. The conditions in Lemma 2.7(1) are equivalent to:

(v) $\operatorname{im} \iota=N^{\perp \perp}$.

(2) Let $\mathcal{D}$ be a full subcategory of $\mathcal{C}$ and $\mathcal{S}_{\mathcal{D}}\left(U^{(I)}\right)$ be the set of all $N \subseteq U^{(I)}$ such that $U^{(I)} / N \in \mathcal{D}$ and $\iota: N \hookrightarrow U^{(I)}$ is strict; in addition, let $\mathcal{S}_{\mathcal{D}}\left(U^{(I)}\right)^{\perp}$ be the set of all $N^{\perp}, N \in \mathcal{S}_{\mathcal{D}}\left(U^{(I)}\right)$. If $W \in \mathcal{C}$ is a cogenerator for $\mathcal{D}$, the Galois connection in Lemma 2.6 induces an order-reversing Galois connection

$$
\mathcal{S}_{\mathcal{D}}\left(U^{(I)}\right) \longleftrightarrow \mathcal{S}_{\mathcal{D}}\left(U^{(I)}\right)^{\perp}: N \mapsto N^{\perp}, \quad \mathfrak{B} \mapsto \mathfrak{B}^{\perp}
$$

which is bijective.

Proof. (1). (i) $\Rightarrow(\mathrm{v})$ : By (2.6), ker $\bullet \varphi \phi_{M}=N^{\perp \perp}$. If (i) holds, then $N^{\perp \perp}=$ $\operatorname{ker} \varphi=\operatorname{ker} \operatorname{coker} \iota=\operatorname{im} \iota . \quad(\mathrm{v}) \Rightarrow(\mathrm{i})$ : If $(\mathrm{v})$ holds, then $\operatorname{ker} \varphi=\operatorname{ker}\left(\varphi \phi_{M}\right)$, hence also by $(6.2),(6.3)$

$$
\operatorname{coim}\left(\varphi \phi_{M}\right)=\operatorname{coim} \varphi=\text { coker ker coker } \iota=\operatorname{coker} \iota=\varphi \text {. }
$$

The canonical decomposition of $\varphi \phi_{M}$ yields

$$
\varphi \phi_{M}=\operatorname{coim}\left(\varphi \phi_{M}\right) g=\varphi g
$$

thus $\phi_{M}=g$ which is a monomorphism since $\mathcal{C}$ is semi-abelian.

(2). If $\iota: N \hookrightarrow U^{(I)}$ is strict, then $\iota=\operatorname{im} \iota$ and by (v), $N=N^{\perp \perp}$ since $W$ is a cogenerator for $\mathcal{D}$, hence $N=\mathfrak{B}^{\perp}$ where $\mathfrak{B}=N^{\perp}$; thus the map $\mathcal{S}_{\mathcal{D}}\left(U^{(I)}\right) \ni N \mapsto$ $N^{\perp} \in \mathcal{S}_{\mathcal{D}}\left(U^{(I)}\right)^{\perp}$ is bijective with inverse $\mathfrak{B} \mapsto \mathfrak{B}^{\perp}$.

Theorem 2.10(2) can be viewed as a generalization of ([50], Sect. 2, Corollary (47)) and ([63], Corollary 1.1) where $\mathcal{D}=\mathcal{C}={ }_{\mathbf{A}}$ Mod. In everything that follows, we make the following

Assumption 2.11. $\mathcal{C}$ is a semi-abelian category and $\mathcal{D}$ is a full subcategory of $\mathcal{C}$, all objects of which are U-cokernels. The two E-modules in Lemma 2.4 are identified under $\sigma$.

2.1.3. $U$-finitely presented objects. Let $\bullet \iota_{j}^{q}: U \hookrightarrow U^{q}$ be the $j$-th injection and $\bullet \pi_{i}^{k}: U^{k} \rightarrow U$ the $i$-th projection. There exists a canonical isomorphism A-linear isomorphism $\operatorname{Hom}_{\mathcal{C}}\left(U^{q}, U^{k}\right) \stackrel{\stackrel{\sim}{\rightarrow}}{q} \mathbf{A}^{k}$ given by

$$
\bullet f \stackrel{\sim}{\rightarrow}\left(R_{i j}\right)_{i, j}, \quad R_{i j} \triangleq \iota_{i}^{q} f \pi_{j}^{k}, \quad f=\sum_{i, j} \pi_{i}^{q} R_{i j} \iota_{j}^{k} .
$$

The matrix $R=\left(R_{i j}\right)_{i, j} \in{ }^{q} \mathbf{A}^{k}$ is written Mat $(f)$. Let $\bullet f: U^{q} \rightarrow U^{k}$ and $\bullet g:$ $U^{k} \rightarrow U^{l}$. Then, Mat $(f g)=\operatorname{Mat}(f) \operatorname{Mat}(g)$. Let $M$ be an object of $\mathcal{C}$ and 
$x \in \operatorname{Hom}_{\mathcal{C}}\left(M, U^{q}\right)=\operatorname{Hom}_{\mathcal{C}}(M, U)^{q}$; then $x=\left(x_{i}\right)_{1 \leq i \leq q}$ is denoted by a row with entries $x_{i}(1 \leq i \leq q)$ and

$$
x f=\left(\sum_{1 \leq i \leq q} x_{i} R_{i j}\right)_{1 \leq j \leq k} \in \operatorname{Hom}_{\mathcal{C}}\left(M, U^{k}\right)=\operatorname{Hom}_{\mathcal{C}}(M, U)^{k}
$$

is the row $x R$, thus the left morphism $\bullet f$ is identified with the right multiplication by the matrix $R=\operatorname{Mat}(f)$; this right multiplication is written $\bullet R$.

Lemma and Definition 2.12. (i) An object $M \in \mathcal{C}$ is called $U$-finitely presented if there exists an exact sequence

$$
U^{q} \stackrel{\bullet f}{\longrightarrow} U^{k} \stackrel{\bullet \varphi}{\longrightarrow} M \longrightarrow 0 .
$$

(ii) In that case, $M=\operatorname{coker} \iota$ where $\iota \triangleq \operatorname{im} f$, and $\iota=\operatorname{im} \iota=\operatorname{ker} \varphi$.

For the proof of (ii), see $\S 6.1 .1$ The morphism $\bullet f$ can also be written $\bullet R$, $R=\operatorname{Mat}(f)$. The $W$-behavior $\mathcal{B} \triangleq N^{\perp}$, identified with $\mathfrak{B}_{W}(M)$, is obtained by applying the functor $\mathfrak{B}_{W}$ to $(2.7)$ which yields the exact sequence of right morphisms

$$
0 \longrightarrow \mathfrak{B}_{W}(M) \stackrel{\mathfrak{B}_{W}(\varphi)}{\longrightarrow} \mathfrak{B}_{W}\left(U^{k}\right) \stackrel{\mathfrak{B}_{W}(\bullet R)}{\longrightarrow} \mathfrak{B}_{W}\left(U^{q}\right)
$$

with the canonical E-linear isomorphisms $\mathfrak{B}_{W}\left(U^{k}\right) \cong{ }^{k} W$ and $\mathfrak{B}_{W}\left(U^{q}\right) \cong{ }^{q} W$; $\mathfrak{B}_{W}(\bullet R)$ is identified with the left multiplication by $R$, written $R \bullet$, and $\mathfrak{B}_{W}(\varphi)$ with the inclusion $\mathfrak{B}_{W}(M) \hookrightarrow^{k} W$, thus setting $\mathfrak{B}_{W}(M)=\mathfrak{B}$ we obtain (in accordance with (2.2))

$$
\mathfrak{B}=\left\{w \in{ }^{k} W: R w=0\right\} \triangleq \operatorname{ker}_{W} R \bullet .
$$

The following generalizes ([50], Sect. 2, Theorem (61)):

TheOrem 2.13. Let $M_{i}=\operatorname{coker} \bullet R_{i}$ be $U$-finitely presented objects of $\mathcal{C}$ $\left(R_{i} \in{ }^{q_{i}} \mathbf{A}^{k}\right)$ and $N_{i}=\operatorname{im} \bullet R_{i}(i=1,2)$, and let $\mathcal{D}$ be a full subcategory of $\mathcal{C}$, all objects of which are $U$-finitely presented.

(i) If there exists a matrix $X \in{ }^{q_{1}} \mathbf{A}^{q_{2}}$ such that $R_{1}=X R_{2}$, then $\operatorname{ker}_{W} R_{2} \bullet \subseteq$ $\operatorname{ker}_{W} R_{1} \bullet$.

(ii) The converse of (i) holds true if $M_{2} \in \mathcal{D}, U$ is projective in $\mathcal{C}$ and $W \in \mathcal{C}$ is a cogenerator for $\mathcal{D}$.

(iii) If $U$ is a generator and the converse of (i) holds true whenever $M_{2} \in \mathcal{D}$, then $W \in \mathcal{C}$ is a cogenerator for $\mathcal{D}$.

Proof. (i) is obvious.

(ii): Since $\mathcal{C}$ is semi-abelian, the morphism $\bullet R_{i}$ can be factored as $\bullet R_{i}=\beta_{i} \omega_{i}$ where $\bullet \beta_{i}: U^{q_{i}} \rightarrow N_{i}$ is an epimorphism and $\omega_{i}: N_{i} \hookrightarrow U^{k}$ is the inclusion. We have $N_{2}^{\perp} \subseteq N_{1}^{\perp}$, thus $N_{1} \subseteq N_{1}^{\perp \perp} \subseteq N_{2}^{\perp \perp}$ by Lemma 2.6, and $N_{2}^{\perp \perp}=N_{2}$ by Theorem 2.10(1) and Lemma and Definition 2.12(ii). Since $N_{1} \subseteq N_{2}$, let $\iota: N_{1} \hookrightarrow N_{2}$ be the inclusion. We have $\bullet R_{1}=\gamma \omega_{2}$ with $\gamma=\beta_{1} \iota: U^{q_{1}} \rightarrow N_{2}$. Since $U$ is projective, so is $U^{q_{1}}$ too and there exists $\bullet \xi: U^{q_{1}} \rightarrow U^{q_{2}}$ such that $\gamma=\xi \beta_{2}$, thus $\gamma \omega_{2}=\xi \beta_{2} \omega_{2}$, i.e. $\bullet R_{1}=\xi \bullet R_{2}$. Therefore, $R_{1}=X R_{2}$ where $X=\operatorname{Mat}(\xi)$. where

(iii): Since $U$ is a generator there is an epimorphism $f=\left(\bullet R_{i}\right)_{i \in I}: U^{(I)} \rightarrow N_{2}^{\perp \perp}$

$$
\text { - } R_{i}: U \stackrel{\iota_{i}}{\hookrightarrow} U^{(I)} \stackrel{f}{\rightarrow} N_{2}^{\perp \perp} \hookrightarrow U^{k}
$$


i.e. $R_{i} \in \mathbf{A}^{k}, \operatorname{im}\left(\bullet R_{i}\right) \subseteq N_{2}^{\perp \perp}$. Then,

$$
\operatorname{ker}_{W}\left(R_{2} \bullet\right)=N_{2}^{\perp}=N_{2}^{\perp \perp \perp} \subseteq \operatorname{im}\left(\bullet R_{i}\right)^{\perp}=\operatorname{ker}_{W}\left(R_{i} \bullet\right) .
$$

If the converse of (i) holds, there are rows $X_{i} \in \mathbf{A}^{q_{2}}$ with $R_{i}=X_{i} R_{2}$ and therefore $\operatorname{im}\left(\bullet R_{i}\right) \subseteq \operatorname{im}\left(\bullet R_{2}\right)=N_{2}$, hence $f=\left(\bullet R_{i}\right)_{i \in I}: U^{(I)} \rightarrow N_{2}$ and $\operatorname{im} f \subseteq N_{2}$. Since $N_{2}^{\perp \perp}$ is a kernel and $f: U^{(I)} \rightarrow N_{2}^{\perp \perp}$, we have $N_{2}^{\perp \perp}=\operatorname{im} f$ and thus also $N_{2}^{\perp \perp} \subseteq N_{2}$; finally, $N_{2}^{\perp \perp}=N_{2}$, thus $W$ is a cogenerator for $\mathcal{D}$ by Theorem 2.10(1).

\subsection{Classical example.}

2.2.1. The category ${ }_{\mathbf{A}}$ Mod. Let $\mathbf{R}$ be a ring and $\mathcal{C}$ the category ${ }_{\mathbf{A}}$ Mod of left $\mathbf{R}$-modules with its left morphisms $x \mapsto(x) f=x f$. Then $U \triangleq \mathbf{R}$ is a projective generator and the map

$$
\mathbf{A} \triangleq \operatorname{Hom}_{\mathbf{R}}(\mathbf{R}, \mathbf{R}) \rightarrow \mathbf{R}, \quad f \mapsto(1) f,
$$

is a ring-isomorphism with inverse $r \mapsto \bullet r$. We identify $\mathbf{A}$ and $\mathbf{R}$ and with this identification,

$$
\operatorname{Hom}_{\mathbf{R}}(\mathbf{R}, M) \rightarrow M: g \mapsto(1) g
$$

is an isomorphism of left $\mathbf{A}$-modules. The category $\mathcal{C}$ is abelian and every object of $\mathcal{C}$ is an A-cokernel ([5], Subsect. I.2.8).

In the present context, Lemma 2.9 can be rephrased as in the corollary below which generalizes a classical result of homological algebra ([39], Corollary (19.7)(1)), obtained when $\mathcal{D}=\mathcal{C}={ }_{\mathrm{A}}$ Mod:

Corollary 2.14. Let $\mathcal{D}$ be a full subcategory of $\mathcal{C}$ such that $\mathbf{A} \in \mathcal{D}$ and $W \in \mathcal{C}$ be a cogenerator for $\mathcal{D}$. Then $W$ is faithful.

Let $N \subseteq \mathbf{A}^{(I)}, M=\mathbf{A}^{(I)} / N$ and $W \in{ }_{\mathbf{A}}$ Mod. According to Pillai and Shankar [53] (see also [63], [72], [64]), $N$ is called $W$-closed if $N=N^{\perp \perp}$. By Theorem 2.10, this means (using the terminology in Definition 2.8) that $W$ is a cogenerator for the subcategory of $\mathcal{C}$ consisting of $M$ alone.

2.2.2. Controllability. The full subcategory of ${ }_{\mathrm{A}} \mathrm{Mod}$, the objects of which are finitely presented, is denoted by $\mathcal{D}={ }_{\mathbf{A}} \mathbf{M o d}^{f p}$ in what follows. Let us recall that a module $M \in{ }_{\mathbf{A}}$ Mod is: (i) torsion-free if whenever $a \in \mathbf{A}$ is right-regular and $a m=0(m \in M)$, then $m=0$; (ii) torsionless if $\mathbf{A}$ is a cogenerator for the subcategory of ${ }_{\mathbf{A}}$ Mod consisting of $M$ alone ([42], 3.4.2). For further reference, the following gathers several results which can be found in the literature, except (ii) and (iv) which slightly generalize ([50], Sect. 2, Lemma and Definition (27)) and ([76], Lemma 1), respectively.

Lemma 2.15. Let $\mathbf{A}$ be a ring and consider the sequence (2.9) below where $R_{1} \in \mathbb{A}^{k}, R_{2} \in{ }^{k} \mathbf{A}^{r}$

$$
\mathbf{A}^{q} \stackrel{\bullet R_{1}}{\longrightarrow} \mathbf{A}^{k} \stackrel{\bullet R_{2}}{\longrightarrow} \mathbf{A}^{r} \rightarrow 0
$$

and let $M_{1}=$ coker $\bullet R_{1}$.

(i) The following conditions are equivalent: (a) the sequence (2.9) is exact at $\mathbf{A}^{r}$, i.e. the map $\bullet R_{2}$ is surjective; (b) $\bullet R_{2}$ is left-invertible; (c) the algebraic transpose $R_{2} \bullet$ of $\bullet R_{2}$ is left-invertible; (d) the matrix $R_{2}$ is left-invertible over $\mathbf{A}$.

(ii) Given $R_{2} \in{ }^{k} \mathbf{A}^{r}$, if $\mathbf{A}$ is a left coherent ring, there exists $R_{1} \in{ }^{q} \mathbf{A}^{k}$ such that the 
sequence (2.9) is exact at $\mathbf{A}^{k}$.

(iii) If the sequence (2.9) is exact at $\mathbf{A}^{k}$, then there exists an embedding $M_{1} \hookrightarrow \mathbf{A}^{r}$; in particular, in that case $M_{1}$ is both torsion-free and torsionless.

(iv) Conversely, given $R_{1} \in{ }^{q} \mathbf{A}^{k}$, if there exists an embedding $M_{1} \hookrightarrow \mathbf{A}^{r}$, there exists a matrix $R_{2} \in{ }^{k} \mathbf{A}^{r}$ such that the sequence (2.9) is exact at $\mathbf{A}^{k}$. Such an embedding exists if the ring $\mathbf{A}$ is right coherent and $M_{1}$ is torsionless, or if the ring $\mathbf{A}$ is semiprime Goldie (in particular, if $\mathbf{A}$ is an Ore domain ${ }^{1}$ ) and $M_{1}$ is torsion-free.

(v) Given $R_{1} \in \in^{q} \mathbf{A}^{k}$, there exists a positive integer $r$ and a matrix $R_{2} \in{ }^{k} \mathbf{A}^{r}$ such that the sequence (2.9) is exact if, and only if, then $M_{1}$ is free.

Proof. (i): Obviously, (b) $\Leftrightarrow(\mathrm{c}) \Leftrightarrow(\mathrm{d}) \Rightarrow(\mathrm{a})$. If (a) holds, $\mathbf{A}^{k} / \mathrm{ker} \bullet R_{2} \cong \mathbf{A}^{r}$ by Noether's first isomorphism theorem, thus ker $\bullet R_{2}$ is a direct summand of $\mathbf{A}^{k}$ and since $\bullet R_{2}$ is surjective, it is left-invertible ([4], Subsect. II.1.9, Corol. 1 of Prop. 15).

(ii): If $\mathbf{A}$ is left coherent, then ker $\bullet R_{2} \subseteq \mathbf{A}^{k}$ is finitely generated ([18], Theorem A.9); a matrix $R_{1}$, the rows of which form a finite generator of ker $\bullet R_{2}$, is universal with respect to $R_{1} R_{2}=0$ ([50], Sect. 2, Lemma and Definition (27)) and then the sequence (2.9) is exact at $\mathbf{A}^{k}$.

(iii): If the sequence (2.9) is exact at $\mathbf{A}^{k}$, there exists an induced map $\left(\bullet R_{2}\right)_{\text {ind }}$ : $M_{1} \rightarrow \mathbf{A}^{r}$ which is an embedding, and then $M_{1}$ is both torsion-free and torsionless since so is $\mathbf{A}^{r}$.

(iv): (a) Consider the exact sequence

$$
\mathbf{A}^{q} \stackrel{\bullet R_{1}}{\longrightarrow} \mathbf{A}^{k} \stackrel{\varphi_{1}}{\longrightarrow} M_{1} \rightarrow 0 .
$$

If there exists an embedding $\bullet \iota: M_{1} \hookrightarrow \mathbf{A}^{r}$, set $\bullet R_{2}=\bullet \varphi_{1} \iota: \mathbf{A}^{k} \rightarrow \mathbf{A}^{r}$. Then the sequence (2.9) is exact at $\mathbf{A}^{k}$.

(b) If $M_{1}$ is torsionless, there exists an embedding $\varepsilon: M_{1} \hookrightarrow \mathbf{A}^{I}$ of $M_{1}$ into a power $\mathbf{A}^{I}$ of $\mathbf{A}$. Set $\bullet \tau=\bullet \varphi_{1} \varepsilon: \mathbf{A}^{k} \rightarrow \mathbf{A}^{I}$; for any $x \in \mathbf{A}^{k}, x \tau$ can be written $x T$ where the matrix $T \in{ }^{k} \mathbf{A}^{I}$ has possibly infinitely many columns. The sequence

$$
\mathbf{A}^{q} \stackrel{\bullet R_{1}}{\longrightarrow} \mathbf{A}^{k} \stackrel{\bullet T}{\longrightarrow} \mathbf{A}^{I}
$$

is exact, thus $R_{1} T=0$. If $\mathbf{A}$ is right coherent, there exists a natural integer $r$ and matrices $R_{2} \in{ }^{k} \mathbf{A}^{r}, D \in{ }^{r} \mathbf{A}^{I}$ such that $R_{1} R_{2}=0, T=R_{2} D$ ([18], Theorem A.6(f)) and the exactness of the sequence (2.10) at $\mathbf{A}^{k}$ implies that of the sequence (2.9) at $\mathbf{A}^{k}$, thus there exists an embedding $M_{1} \hookrightarrow \mathbf{A}^{r}$ by (iii).

(c) The same holds if $\mathbf{A}$ is semiprime Goldie and $M$ is torsion-free ([42], Prop. 3.4.7), and any Ore domain is a semiprime Goldie ring.

(v): (a) Assume $M_{1}$ is free of rank $r$. The above monomorphism $\iota: M_{1} \hookrightarrow \mathbf{A}^{r}$ is then an isomorphism, thus $\bullet R_{2}=\bullet \varphi_{1} \iota$ is an epimorphism and the sequence (2.9) is exact. (b) Conversely, if the sequence (2.9) is exact, (iii) holds and $\left(\bullet R_{2}\right)_{\text {ind }}$ is an isomorphism, thus $M_{1} \cong \mathbf{A}^{r}$ is free.

We consider now the exact sequence (2.7) with $U=\mathbf{A}$ and the $W$-behavior $\mathfrak{B}_{W}(M)=\mathfrak{B}$ defined by (2.8). As shown in [69], ([54], Sect. 6.6), [58], [73], [53], [74], [63], where various kinds of rings and signal spaces are considered, the "behavioral controllability" is linked to the existence of an "image representation" of $\mathfrak{B}_{W}(M)$, i.e. to the existence of matrix $S \in{ }^{k} \mathbf{A}^{r}$ such that

$$
\mathfrak{B}_{W}(M)=\operatorname{im}_{W} S \bullet \triangleq\left\{\in{ }^{k} W: \exists \xi \in{ }^{r} W, w=S \xi\right\} .
$$

\footnotetext{
${ }^{1}$ In this paper, the expressions Ore, Noetherian, coherent, etc. are used for two-sided Ore, twosided Noetherian, two-sided coherent, etc.
} 
When $\mathfrak{B}_{W}(M)$ has an image representation of the form $(2.11)$ where $S \bullet:{ }^{r} W \rightarrow$ $\mathfrak{B}_{W}(M)$ is injective (thus bijective), this image representation is said to be "observable" since then the "latent variable" $\xi$ is uniquely determined by the "manifest variable" $w$ ([54], Sect. 5.3), and $\mathfrak{B}_{W}(M)$ is said to be strongly controllable [59], [71].

On the other hand, according to Fliess and Mounier [29], [48], [49], a linear system is a finitely presented left A-module $M$. Such a system is said to be free controllable (resp. torsion-free controllable) if the module $M$ is free (resp. torsion-free). In accordance with Zerz [76], a linear system $M$ is said to be torsionless controllable if the module $M$ is torsionless. A free controllable linear system $M$ is also said to be flat and a basis of the free module $M$ is called a flat output [30].

The consistency between the two above points of view has been widely studied when the A-module $W$ is injective (see, e.g., [53], [17], [71], [72]). It is clarified below without this assumption.

THEOREM 2.16. (1) If $\bullet S: \mathbf{A}^{k} \rightarrow \mathbf{A}^{r}$ is left-invertible, then the image representation (2.11) is observable, and the converse holds true if $W$ is a cogenerator for $\mathcal{D}={ }_{\mathbf{A}} \operatorname{Mod}^{f p}$.

(2). (i) If $M=$ coker $\bullet R$ is a free module, then $\mathfrak{B}_{W}(M)$ has an observable image representation. (ii) Conversely, if $W$ is cogenerator for $\mathcal{D}$ in $\mathbf{A} \mathbf{M o d}$ and $\mathfrak{B}_{W}(M)$ has an observable image representation, then $M$ is free.

(3). (i') Assume that the ring $\mathbf{A}$ is right coherent (resp. semiprime Goldie) and that the $\mathbf{A}$-module $W$ is faithful. If $M$ is torsionless (resp. torsion-free), then there exists a matrix $S$ over $\mathbf{A}$ such that $\mathfrak{B}_{W}(M)=\left(\operatorname{im}_{W} S \bullet\right)^{\perp \perp}$. (ii') Conversely, if $W$ is a cogenerator for $\mathcal{D}$, then the equality $\mathfrak{B}_{W}(M)=\left(\operatorname{im}_{W} S \bullet\right)^{\perp \perp}$ implies that $M$ is both torsionless and torsion-free.

Proof. (1) If $\bullet S: \mathbf{A}^{k} \rightarrow \mathbf{A}^{r}$ is left-invertible, then the image representation (2.11) is obviously observable. Conversely, consider the exact sequence

$$
\mathbf{A}^{k} \stackrel{\bullet S}{\longrightarrow} \mathbf{A}^{r} \stackrel{\psi}{\longrightarrow} N \rightarrow 0
$$

which yields (through the functor $\mathfrak{B}_{W}(\bullet)$ ) the exact sequence

$$
0 \longrightarrow \mathfrak{B}_{W}(N) \longrightarrow{ }^{r} W \stackrel{S \bullet}{\longrightarrow}{ }^{k} W .
$$

If $S \bullet:{ }^{r} W \rightarrow \mathfrak{B}_{W}(M)$ is injective, this implies $\mathfrak{B}_{W}(N)=0$, and finally $N=0$ if in addition $W$ is a cogenerator for $\mathcal{D}$. Therefore, $\bullet S$ is surjective, thus left-invertible by Lemma 2.15(i).

(2). (i) is obvious.

(ii): Assuming that $\mathfrak{B}_{W}(M)$ has an observable image representation, (2.11) holds with $\bullet S$ left-invertible by (1), thus the sequence

$$
0 \longrightarrow{ }^{r} W \stackrel{S \bullet}{\longrightarrow}{ }^{k} W \stackrel{R \bullet}{\longrightarrow}{ }^{q} W
$$

is exact in $\operatorname{Mod}_{\mathbf{E}}$ and there exists a matrix $T \in{ }^{r} \mathbf{A}^{k}$ such that $T S=I_{r}$. This equality proves that $\bullet S$ is surjective and the exactness of (2.12) proves that $S \bullet$ is injective and $R S \bullet=0$, thus $R S=0$ since $W$ is a cogenerator for $\mathcal{D}$. Therefore, $\mathbf{A}^{q} R \subseteq$ ker $\bullet S$ and there exists a map $\bullet f$ such that the diagram below is commutative

$$
\begin{array}{lll}
\mathbf{A}^{k} & \stackrel{\bullet S}{\longrightarrow} & \mathbf{A}^{r} \\
\downarrow \varphi & f \nearrow & \\
M & &
\end{array}
$$


where $\bullet \varphi: \mathbf{A}^{k} \rightarrow M=\mathbf{A}^{k} / \mathbf{A}^{q} R \in{ }_{\mathbf{A}} \mathbf{M o d}^{f p}$ is the canonical epimorphism and - $f: M \rightarrow \mathbf{A}^{r}$ is an epimorphism too since so is $\bullet S$. Thus $\mathfrak{B}_{W}(M)=\operatorname{ker}_{W}(R \bullet)=$ $\operatorname{im}_{W}(S \bullet) \cong r$ (by exactness of $(2.12)$ ) and since the functor $\mathfrak{B}_{W}$ is left exact, the surjectivity of $\bullet f$ implies the exactness of

$$
0 \longrightarrow{ }^{r} W^{\stackrel{\mathfrak{B}_{W}(\bullet f)}{\longrightarrow}} \mathfrak{B}_{W}(M),
$$

thus $\mathfrak{B}_{W}(\bullet f):{ }^{r} W \rightarrow \mathfrak{B}_{W}(M)$ is an embedding. Therefore, $\mathfrak{B}_{W}(\bullet f)$ is an isomor-

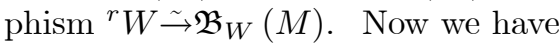

$$
I d_{\mathbf{A}^{r}}=\bullet T S=\bullet T \varphi f=g f, \quad g \triangleq \bullet T \varphi: \mathbf{A}^{r} \rightarrow M .
$$

Thus $\bullet f=\bullet f g f$, hence $\mathfrak{B}_{W}(\bullet f) \bullet=\mathfrak{B}_{W}(\bullet f g) \mathfrak{B}_{W}(\bullet f) \bullet$ which implies $\mathfrak{B}_{W}(\bullet f g)=$ $I d_{r}$ for $\mathfrak{B}_{W}(\bullet f)$ is invertible. Since $W$ is a cogenerator for $\mathcal{D}$, this implies $\bullet f g=$ $I d_{\mathbf{A}^{r}}$, thus $\bullet f$ is injective; finally, $\bullet f$ an isomorphism $M \stackrel{\sim}{\rightarrow} \mathbf{A}^{r}$, and $M$ is free.

(3). (i'): By Lemma 2.15(iv), there exists a matrix $S$ such that the sequence

$$
\mathbf{A}^{q} \stackrel{\bullet R}{\longrightarrow} \mathbf{A}^{k} \stackrel{\bullet S}{\longrightarrow} \mathbf{A}^{r} \rightarrow 0
$$

is exact at $\mathbf{A}^{k}$, i.e. $\operatorname{im} \bullet R=\operatorname{ker} \bullet S$ which implies $(\operatorname{im} \bullet R)^{\perp}=(\operatorname{ker} \bullet S)^{\perp}$. We have $(\mathrm{im} \bullet R)^{\perp}=\operatorname{ker}_{W} R \bullet$. Let $r \in \operatorname{ker} \bullet S$; then $r S=0$ which implies that $r S \xi=0$ for any $\xi \in W^{r}$, thus $\operatorname{ker} \bullet S \subseteq\left(\operatorname{im}_{W} S \bullet\right)^{\perp}$. If $W$ is faithful, we conclude that

$$
\operatorname{ker} \bullet S=\left(\operatorname{im}_{W} S \bullet\right)^{\perp}
$$

thus $\operatorname{ker}_{W} R \bullet=\left(\operatorname{im}_{W} S \bullet\right)^{\perp \perp}$.

(ii'): Assuming that $\operatorname{ker}_{W} R \bullet=\left(\operatorname{im}_{W} S \bullet\right)^{\perp \perp}$ and $W$ is a cogenerator for ${ }_{\mathbf{A}} \mathbf{M o d}^{f p}$ in ${ }_{\mathrm{A}}$ Mod, we have $\left(\operatorname{ker}_{W} R \bullet\right)^{\perp}=\left(\operatorname{im}_{W} S \bullet\right)^{\perp}$, thus $\operatorname{im} \bullet R=\left(\operatorname{im}_{W} S \bullet\right)^{\perp}=\operatorname{ker} \bullet S$ by Theorem 2.10, Corollary 2.14 and (2.13). The conclusion follows from Lemma 2.15(iii) with $R_{1}=R, R_{2}=S$.

REMARK 2.17. (i) A non-trivial proof of Statement (2)(ii) of the above theorem is needed because the $\mathbf{A}$-module $W$ is not assumed to be injective, therefore the exactness of a sequence

$$
\mathfrak{B}_{W}\left(M_{3}\right) \stackrel{R_{2} \bullet}{\longrightarrow} \mathfrak{B}_{W}\left(M_{2}\right) \stackrel{R_{1} \bullet}{\longrightarrow} \mathfrak{B}_{W}\left(M_{1}\right)
$$

in $\operatorname{Mod}_{\mathbf{E}}$ does not imply the exactness of

$$
M_{1} \stackrel{\bullet R_{1}}{\longrightarrow} M_{2} \stackrel{\bullet R_{2}}{\longrightarrow} M_{3}
$$

in ${ }_{\mathbf{A}}$ Mod, despite Corollary and Definition 2.8(ii); compare with ([56], Sect. 3) and ([17], Theorem 3(6)). The reason for this is that the sequence (2.14) is not necessarily exact in $\mathfrak{B}_{W}(\mathcal{D})$, for this category is not full in $\mathbf{M o d}_{\mathbf{E}}$. According to ([50], Sect. 2, Duality Theorem (56)), $\mathfrak{B}_{W}(\mathcal{D})$ is full in $\boldsymbol{M}_{\mathbf{E}}$ if $W$ is a large injective cogenerator. (ii) To specify the above item, recall that a cogenerator in ${ }_{\mathbf{A}}$ Mod need not be injective.

(a) An example of non-injective cogenerator is given in ([39], §19, Exercise 20).

(b) Let us give a second example: let $\mathbf{A}$ be a ring, ${ }_{\mathbf{A}} W_{1}$ an injective cogenerator and $W_{2} \neq 0$ a non-injective left $\mathbf{A}$-module, e.g. $\mathbf{A}=\mathbb{C}[d / d t], W_{1}=\mathcal{E}(\mathbb{R}), W_{2}=\mathcal{D}(\mathbb{R})$. Then $W \triangleq W_{1} \oplus W_{2}$ is a cogenerator which is a non-injective left $\mathbf{A}$-module by ([39], Prop. 3.4). 


\subsection{Topological modules and behaviors.}

2.3.1. The category A TMod. In everything that follows, we make the following

Assumption 2.18. $\mathbf{k}$ is the field of real or complex numbers and all algebras are associative, unitary and defined over $\mathbf{k}$.

Let $\mathbf{A}$ be an algebra, $W$ a $\mathbf{k}$-vector space and a nondegenerate bilinear form ([7], Subsect. II.6.1)

$$
\langle-,-\rangle: \mathbf{A} \times W \rightarrow \mathbf{k} .
$$

We also consider the nondegenerate bilinear form

$$
\langle-,-\rangle^{\tilde{2}}: W \times \mathbf{A} \rightarrow \mathbf{k}
$$

defined by $\langle w, a\rangle^{\tilde{}}=\langle a, w\rangle$ for any $a \in \mathbf{A}$ and $w \in W$. The weak topology $\sigma(\mathbf{A}, W)$ (resp. $\sigma(W, \mathbf{A})$ ) is the coarsest topology in $\mathbf{A}$ (resp. in $W$ ) which makes continuous all linear forms $a \mapsto\langle a, w\rangle, w \in W$ (resp. $w \mapsto\langle a, w\rangle, a \in \mathbf{A}$ ). The following facts are classical ([7], Subsect. II.6.2): the space $W$ is canonically isomorphic to, and identified with, the topological dual $\mathbf{A}^{\prime}$ of $(\mathbf{A}, \sigma(\mathbf{A}, W))$, i.e. of $\mathbf{A}$ endowed with the topology $\sigma(\mathbf{A}, W)$; this statement is also valid with $W$ and $\mathbf{A}$ interchanged; $(\mathbf{A}, \sigma(\mathbf{A}, W))$ and $(W, \sigma(W, \mathbf{A}))$ (respectively written $\mathbf{A}$ and $W$ in what follows, for short) are locally convex topological vector spaces (LCTVS's) and Hausdorff.

A left A-module $M$ is called topological if it is endowed with a structure of LCTVS and if all maps $\mathbf{A} \rightarrow M: a \mapsto a m, m \in M$, are continuous; a topological right $\mathbf{A}$ module $M$ is similarly defined. (Note that the definition of a topological module over a topological ring is different ([6], Subsect. III.6.6).) The algebra A may be itself a topological left or right $\mathbf{A}$-module. The algebraic dual $\mathbf{A}^{*}=\operatorname{Hom}_{\mathbf{k}}(\mathbf{A}, \mathbf{k})$ is a left and right $\mathbf{A}$-module via

$$
(x) a \alpha=(x a) \alpha \quad \text { and }(\alpha a) x=\alpha(a x), \quad \alpha \in \mathbf{A}^{*}, a, x \in \mathbf{A}
$$

(using Notation 2.1(i)). In general, $\mathbf{A}^{\prime}=W$ is not an $\mathbf{A}$-submodule of $\mathbf{A}^{*}$ and when this happens is characterized below:

Lemma 2.19. (1) The following conditions are equivalent:

(i) The left module $\mathbf{A} \mathbf{A}$ is topological, i.e. all maps $a \mapsto a b(b \in \mathbf{A})$ are continuous.

(ii) $W$ is a left $\mathbf{A}$-module with $\langle a, b w\rangle=\langle a b, w\rangle$ for all $a, b \in \mathbf{A}$ and $w \in W$.

Then also all maps $W \rightarrow W: w \mapsto b w(b \in \mathbf{A})$ are continuous.

(2) The following properties are equivalent:

(i') The right module $\mathbf{A}_{\mathbf{A}}$ is topological.

(ii') $W$ is a right $\mathbf{A}$-module with $\langle w a, b\rangle^{\sim}=\langle w, a b\rangle^{\sim}$ for all $a, b \in \mathbf{A}$.

Then also all maps $W \rightarrow W: w \mapsto w b(b \in \mathbf{A})$ are continuous.

(3) If the conditions of (1) hold, then those of (2) are equivalent to:

(iii') The left module $\mathbf{A} W$ is topological.

Proof. (1). (i) $\Rightarrow$ (ii): If $a \mapsto a b$ is continuous, then so is $b \alpha: a \mapsto a(b \alpha)=(a b) \alpha$ for any $\alpha \in \mathbf{A}^{\prime}=W$ with the identification $W \ni w \mapsto\langle-, w\rangle \in \mathbf{A}^{\prime}$. Hence $\mathbf{A}^{\prime}=W$ is an $\mathbf{A}$-submodule of $\mathbf{A}^{*}$. (ii) $\Rightarrow$ (i): If (ii) holds, then $b w \in W$ for any $b \in \mathbf{A}, w \in W$, therefore the map $\mathbf{A} \ni a \mapsto\langle a, b w\rangle=\langle a b, w\rangle \in \mathbf{k}$ is continuous which implies that so is the map $a \mapsto a b$. The maps $w \mapsto\langle a, b w\rangle$ are continuous by the above equality. (2) is shown like (1). 
(3). If (ii) holds, the following conditions are equivalent: all maps $b \mapsto b w$ are continuous, i.e. (iii') holds; all maps $b \mapsto\langle a, b w\rangle=\langle a b, w\rangle$ are continuous; all maps $b \mapsto a b$ are continuous, i.e. (i') holds.

Definition 2.20. Assuming that all conditions of Lemma 2.19 are satisfied, the category of topological left $\mathbf{A}$-modules and continuous linear maps is called the category of topological left A-modules and written A TMod.

LEMMA 2.21. A TMod is a semi-abelian category with arbitrary products and coproducts and with projective generator $\mathbf{A}$.

Proof. (a) The category $\mathcal{C}={ }_{\mathbf{A}} \mathbf{T}$ Tod is a subcategory of ${ }_{\mathbf{A}} \mathbf{M o d}$; $\mathcal{C}$ is obviously additive and has kernels and cokernels which are the algebraic ones endowed with the induced and coinduced topology, respectively, thus $\mathcal{C}$ is preabelian. Let $\bullet f: X \rightarrow Y$ be a morphism of $\mathcal{C}$ and $f_{\text {ind }}$ be its induced morphism in ${ }_{\mathbf{A}} \mathbf{M o d}$. Then $f_{\text {ind }}$ is bijective (since $\mathbf{A}$ Mod is abelian) and continuous by definition of the topologies of $\operatorname{coim} f$ and of $\operatorname{im} f$. Therefore, $f_{\text {ind }}$ is the induced morphism of $f$ in $\mathcal{C}$. Since $f_{\text {ind }}$ is both a monomorphism and an epimorphism, the preabelian category $\mathcal{C}$ is semi-abelian. Last, $\mathcal{C}$ has arbitrary products and coproducts which are the algebraic ones endowed with the product topology and the locally convex direct sum topology, respectively ([7], Subsect. II.4.5, Definition 2).

(b) Let $M \in \mathcal{C}$. There exists an A-linear surjection $\bullet \varphi: \mathbf{A}^{(I)} \rightarrow M$. Let $\bullet \iota: \mathbf{A} \hookrightarrow \mathbf{A}^{(I)}$ be the canonical injection. Let $\bullet f=\bullet \bullet \varphi: \mathbf{A} \rightarrow M$; for any $a \in \mathbf{A}$, (a) $f=a m$ where $m=(1) f$, thus $f$ is continuous since the module $M$ is topological. Therefore, $\varphi$ is continuous (if $\mathbf{A}^{(I)}$ is endowed with the locally convex direct sum topology) according to ([7], Subsect. II.4.5, Prop. 6). This proves that $\mathbf{A}$ is a generator of $\mathcal{C}$.

(c) Let $\bullet f: \mathbf{A} \rightarrow M$ and $\bullet g: M_{1} \rightarrow M$ be respectively a morphism and an epimorphism of $\mathcal{C}$. Since $\bullet f$ and $\bullet g$ are respectively a morphism and an epimorphism of ${ }_{\mathbf{A}}$ Mod, there exists a morphism $\bullet h: \mathbf{A} \rightarrow M_{1}$ of ${ }_{\mathbf{A}} \mathbf{M o d}$ such that $\bullet f=\bullet h g$. Since $M_{1}$ is topological, $\bullet h$ is continuous, i.e. is a morphism of $\mathcal{C}$, thus $\mathbf{A}$ is projective in $\mathcal{C}$.

2.3.2. Cogenerators for topological modules. For $X, Y \in \mathbf{A}_{\mathbf{A}} \mathbf{T M o d}$, we denote by $\operatorname{Hom}_{\mathbf{A}, c}(X, Y)$ the $\mathbf{k}$-vector space consisting of all continuous A-linear maps $X \rightarrow Y$. Endowed with the topology of pointwise convergence, $\operatorname{Hom}_{\mathbf{A}, c}(X, Y)$ is an LCTVS; in particular we have the additive left exact contravariant functor

$$
\mathfrak{B}_{W}:{ }_{\mathbf{A}} \mathbf{T M o d} \rightarrow \operatorname{Mod}_{\mathbf{E}}: M \mapsto \mathfrak{B}_{W} \triangleq \operatorname{Hom}_{\mathbf{A}, c}(M, W), \quad \mathbf{E} \triangleq \operatorname{Hom}_{\mathbf{A}, c}(W, W) .
$$

The image $\mathfrak{B}_{W}$ (A TMod) is called the class of all (topological) $W$-behaviors. All results in Subsect. 2.1 are valid, in particular Theorems 2.10 and 2.13.

REMARK 2.22. Let $M \in \mathcal{C}$. By Lemma 2.21, there exists a set of indices $I$ such that $M$ is a factor of $\mathbf{A}^{(I)}$, i.e. there exists a continuous $\mathbf{A}$-linear surjection $\varphi: \mathbf{A}^{(I)} \rightarrow M$. There exists a continuous bijection $\varphi_{\text {ind }}: \mathbf{A}^{(I)} / N \rightarrow M$ where $N=\operatorname{ker} \varphi$. But $\varphi_{\text {ind }}^{-1}$ is not continuous in general, i.e. is not an isomorphism of $\mathcal{C}$, thus $\mathbf{A}^{(I)} / N$ and $M$ cannot be identified; then, $M$ is not an A-cokernel.

Let $I$ be a set of indices. The nondegenerate form $\langle-,-\rangle$ is extended to the nondegenerate form

$$
\langle-,-\rangle: \mathbf{A}^{(I)} \times{ }^{I} W:(x, w) \mapsto \sum_{i \in I}\left\langle x_{i}, w_{i}\right\rangle
$$

where all terms of the sum are zero except a finite number of them. The locally convex topologies induced by this form in ${ }^{I} W$ and $\mathbf{A}^{(I)}$ are respectively the product 
topology $\sigma\left({ }^{I} W, \mathbf{A}^{(I)}\right)=\prod_{i \in I} \sigma(W, \mathbf{A})([7]$, Subsect. II.6.6, Prop. 8) and the topology $\sigma\left(\mathbf{A}^{(I)},{ }^{I} W\right)$. The latter coincides with the locally convex direct sum topology $\bigoplus_{i \in I} \sigma(\mathbf{A}, W)$ if, and only if $I$ is finite ([61], Chap. IV, Exercise 8). In the sequel, $\mathbf{A}^{(I)}$ is endowed with the topology $\sigma\left(\mathbf{A}^{(I)},{ }^{I} W\right)$ and $I$ may be assumed to be finite,

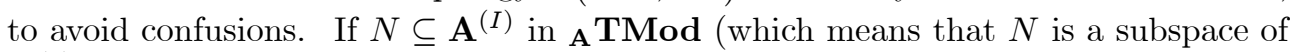
$\left.\mathbf{A}^{(I)}\right)$, its polar $N^{0} \subseteq{ }^{I} \mathbf{A}$ is defined as usual:

$$
N^{0}=\left\{w \in{ }^{I} W:\langle N, w\rangle=0\right\}=\bigcap_{x \in N} \operatorname{ker}\langle x,-\rangle
$$

and $N^{0}$ is closed in ${ }^{I} W$. According to the bipolar theorem ([7], Subsect. II.6.3), $N^{00}=\bar{N}$ where $\bar{N}$ denotes the closure of $N$ in $\mathbf{A}^{(I)}$.

Theorem 2.23. (i) Let $N \subseteq \mathbf{A}^{(I)}$; then

$$
N^{\perp}=N^{0} \quad \text { and } \quad N^{\perp \perp}=N^{00}=\bar{N} .
$$

(ii) Let $M=\mathbf{A}^{(I)} / N$ be an $\mathbf{A}$-cokernel in $\mathcal{C}={ }_{\mathbf{A}} \mathbf{T M o d}$; then $M$ is Hausdorff if, and only if $N=N^{\perp \perp}$.

(iii) Let $\mathcal{D}$ be a full subcategory of $\mathcal{C}$, all objects of which are $\mathbf{A}$-cokernels. Then $W \in \mathcal{C}$ is a cogenerator for $\mathcal{D}$ if, and only if all objects $M=\mathbf{A}^{(I)} / N$ of $\mathcal{D}$ are Hausdorff.

Proof. (i): (a) The identity

$$
\langle a x, w\rangle=\langle a, x w\rangle, a \in \mathbf{A}, x \in \mathbf{A}^{(I)}, w \in{ }^{I} W
$$

holds. By definition, $w \in N^{\perp}$ if, and only if for any $x=\left(x_{i}\right)_{i \in I} \in \mathbf{A}^{(I)}, x w \triangleq$ $\sum_{i \in I} x_{i} w_{i}=0$. Since $\langle-,-\rangle$ is nondegenerate, the last equality is equivalent to $0=\langle a, x w\rangle=\langle a x, w\rangle$ for any $a \in \mathbf{A}$, thus to $\langle x, w\rangle=0$. Thus $N^{\perp}=N^{0}$. (b) Since the module $\mathbf{A}_{\mathbf{A}}$ is topological, the maps $\mathbf{A} \rightarrow \mathbf{A}: x \mapsto a x$ and $\mathbf{A}^{(I)} \rightarrow \mathbf{A}^{(I)}: x \rightarrow a x$ $(a \in \mathbf{A})$ are continuous ([7], Subsect. II.4.5). This implies that $a \bar{N} \subseteq \overline{a N} \subseteq \bar{N}$ and that $\bar{N}$ is a left $\mathbf{A}$-submodule of $\mathbf{A}^{(I)}$. Now, $x \in N^{\perp \perp}$ if, and only if for any $w \in N^{\perp}=N^{0}, x w=0$. The last equality holds if, and only if for all $a \in \mathbf{A}$, $0=\langle a, x w\rangle=\langle a x, w\rangle$, i.e. $a x \in N^{00}=\bar{N}$. Since $\bar{N}$ is a left $\mathbf{A}$-submodule of $\mathbf{A}^{(I)}$, this is equivalent to $x \in \bar{N}$. 2.10.

(i) implies (ii) which implies (iii) by Lemma and Definition 2.12(ii), and Theorem

REMARK 2.24. (i) Theorem 2.23 is a generalization of ([28], Theorem 4.1) and of ([33], Theorem 3.4) where $W=\mathcal{E}(\mathbb{R}), \mathbf{A}$ is the convolution algebra $\left(W^{\prime}, \star\right)$ and $\mathbf{A}$ acts on $W$ via the convolution product.

(ii) Let $W$ be an LCTVS and $\mathbf{A} \subseteq W^{\prime}$. Then, the restriction to $\mathbf{A} \times W$ of the canonical bilinear form $\langle-,-\rangle: W^{\prime} \times W \rightarrow \mathbf{k}$ is possibly degenerate; the topology $\sigma(\mathbf{A}, W)$ is Hausdorff (since it is induced from $\sigma\left(W^{\prime}, W\right)$ ) but $\sigma(W, \mathbf{A})$ is not in general ([7], Subsect. II.6.1, Prop. 1). Therefore, the statements of Theorem 2.23 are no longer valid. This situation is further investigated in the two next sections where convolution systems are considered.

\section{Differential systems with lumped shifts over Lie groups.}

3.1. Actions over a Lie group and convolution algebra. In everything that follows, we'll use the following

Notation 3.1. G is a Lie group with Lie algebra $\mathfrak{g}, W=\mathcal{E}(\mathbf{G})$ is the space all $\mathbf{k}$-valued indefinitely differentiable functions on $\mathbf{G}$, and its topological dual $W^{\prime}$ is the 
space of all $\mathbf{k}$-valued distributions with compact support included in $\mathbf{G}$ (Appendix 2, §6.2.2).

The group $\mathbf{G}$ continuously acts on $W$ via a left and a right action, both of them denoted by $\circ$, and defined by

(i) $(y)(a \circ \varphi)=(y a) \varphi \quad$ (left action of $\mathbf{G}$ on a left map, which is a right shift),

(ii) $(\varphi \circ a)(x)=\varphi($ ax $) \quad$ (right action of $\mathbf{G}$ on a right map, which is a left shift),

for any $x, y, a \in \mathbf{G}$ and $\varphi \in W$. By (Appendix 2, (6.12)), this yields for any $S, T \in W^{\prime}$

$$
\langle S, a \circ \varphi\rangle=\left\langle S \star \delta_{a}, \varphi\right\rangle, \quad\langle\varphi \circ a, T\rangle^{\sim}=\left\langle\varphi, \delta_{a} \star T\right\rangle^{\sim}
$$

(see (6.9) for the latter notation which is consistent with (2.15)).

These actions induce respectively a right and a left action on $W^{\prime}$, both of them also denoted by $\circ$, according to

$$
\langle S \circ a, \varphi\rangle=\langle S, a \circ \varphi\rangle, \quad\langle\varphi, a \circ T\rangle^{\tilde{}}=\langle\varphi \circ a, T\rangle^{\sim}
$$

and from the above

$$
S \circ a=S \star \delta_{a}, \quad a \circ T=\delta_{a} \star T .
$$

We also define a left and a right action of $W^{\prime}$ on $W$, both of them denoted by $\circ$, respectively by

$$
\begin{aligned}
& S \circ: W \rightarrow W:(S \circ \varphi)(x)=\langle S, \varphi \circ x\rangle \\
& \circ S: W \rightarrow W:(x)(\varphi \circ S)=\langle x \circ \varphi, S\rangle^{\sim}
\end{aligned}
$$

for any $\varphi \in W$ and $S \in W^{\prime}$. As a consequence of (Appendix 2, (6.13)) one easily obtains

$$
\varphi \circ S=\check{S} \star \varphi, \quad S \circ \varphi=\varphi \star \check{\Delta}^{-1} \check{S}
$$

where $\Delta$ is the modulus function, $\check{\varphi}(y)=\varphi\left(y^{-1}\right)$ and $\langle\check{S}, \varphi\rangle=\langle S, \check{\varphi}\rangle$ for any $y \in \mathbf{G}$, $\varphi \in W$ and $S \in W^{\prime}$. We have the following:

LEMma 3.2. (i) The equality

$$
\langle T, S \circ \varphi\rangle=\langle\varphi \circ T, S\rangle^{\sim}
$$

holds for any $S, T \in W^{\prime}$ and $\varphi \in W$.

(ii) Both actions o of $W^{\prime}$ on $W$ induce one bilinear law of composition $\circ: W^{\prime} \times W^{\prime} \rightarrow$ $W^{\prime}$ according to

$$
\langle T \circ S, \varphi\rangle=\langle T, S \circ \varphi\rangle, \quad\langle\varphi, T \circ S\rangle^{\sim}=\langle\varphi \circ T, S\rangle^{\sim}
$$

and this law of composition is the usual convolution product $\star$.

(iii) For any $S, T \in W^{\prime}$ and $\varphi \in W$,

$$
(T \circ S) \circ \varphi=T \circ(S \circ \varphi), \quad \varphi \circ(T \circ S)=(\varphi \circ T) \circ S,
$$

i.e. $W$ is a left and right $W^{\prime}$-module.

Proof. (i): We have

$$
\langle T, S \circ \varphi\rangle=\int_{\mathbf{G}} d T(x) \int_{\mathbf{G}} d S(y)(\varphi \circ x)(y)=\int_{\mathbf{G}} d T(x) \int_{\mathbf{G}} d S(y) \varphi(x y)
$$


thus

$$
\langle T, S \circ \varphi\rangle=\langle T \star S, \varphi\rangle .
$$

Similarly (denoting $\varphi$ as a left map)

$$
\langle\varphi \circ T, S\rangle^{\sim}=\int_{\mathbf{G}} d S(y) \int_{\mathbf{G}} d T(x)(x)(y \circ \varphi)=\int_{\mathbf{G}} d S(y) \int_{\mathbf{G}} d T(x)(x y) \varphi
$$

thus

$$
\langle\varphi \circ T, S\rangle^{\tilde{}}=\langle T \star S, \varphi\rangle .
$$

(ii): This is an obvious consequence of (3.6) and (3.7).

(iii): By (3.2), (3.4) we get

$$
\langle U, T \circ(S \circ \varphi)\rangle=\langle U \star T * S, \varphi\rangle=\langle U,(T \star S) \circ \varphi\rangle=\langle U,(T \circ S) \circ \varphi\rangle
$$

which proves the first equality of (3.5). The second one is likewise deduced from (3.3) and (3.4).

Definition 3.3. A convolution algebra is a subalgebra of $\left(W^{\prime}, \circ\right)$.

3.2. Fundamental principle and divisibility. Let $\mathbf{B}$ be a convolution algebra. The restriction to $\mathbf{B} \times W$ of the action $\circ: W^{\prime} \times W \rightarrow W$ and that of the canonical bilinear form $\langle-,-\rangle: W^{\prime} \times W \rightarrow \mathbf{k}$ are still denoted by $\circ$ and $\langle-,-\rangle$, respectively. In general the latter is degenerate, then the topology $\sigma(\mathbf{B}, W)$ of $\mathbf{B}$ is Hausdorff but not the topology $\sigma(W, \mathbf{B})$ of $W$ (see Remark 2.24(ii)). Therefore, the theory in Subsect. 2.3 cannot be directly applied.

Example 3.4. Consider the case when $\mathbf{B}=\mathrm{U}_{\mathbf{k}}(\mathfrak{g})$ (Appendix 2, §6.2.4); we have $\mathbf{B}=\bigoplus_{\alpha \in \mathbb{N}^{n}} D_{\xi}^{\alpha} \delta_{e}$ (where $\mathbb{N}$ is the set of non-negative integers and the notation in §6.2.2 is used); the polar $\mathbf{A}^{o}$ of $\mathbf{A}$ is given by

$$
\mathbf{A}^{o}=\left\{w \in W: \forall \alpha \in \mathbb{N}^{n}, D_{\xi}^{\alpha} w(e)=0\right\}
$$

thus $\mathbf{A}^{o} \neq 0$ (except in the trivial case $\mathbf{G}=\{e\}$ ). We have

$$
\mathbf{A}^{\perp}=\left\{w \in W: \forall \alpha \in \mathbb{N}^{n}, D_{\xi}^{\alpha} \delta_{e} \circ w=0\right\}=0
$$

where $D_{\xi}^{\alpha} \delta_{e} \circ w=D_{\xi}^{\alpha} w$, thus $\mathbf{A}^{o} \neq \mathbf{A}^{\perp}$ and Statement (ii) of Theorem 2.23 does not hold.

Let $I$ be a set of indices; the canonical bilinear form $\langle-,-\rangle: \mathbf{B} \times W \rightarrow \mathbf{k}$ and the action $\circ: \mathbf{B} \times W \rightarrow W$ are extended to $\mathbf{B}^{(I)} \times{ }^{I} W$ according to

$$
\begin{aligned}
\langle-,-\rangle & : \mathbf{B}^{(I)} \times{ }^{I} W:(T, w) \\
\circ & : \sum_{i \in I}\left\langle T_{i}, w_{i}\right\rangle \\
{ }^{(I)} \times{ }^{I} W:(T, w) & \mapsto \sum_{i \in I} T_{i} \circ w_{i} .
\end{aligned}
$$

As usual, the orthogonal space with respect to $\langle-,-\rangle$, i.e. the polar, is denoted by $(-)^{0}$, and that with respect to $\circ$ by $(-)^{\perp}$.

The notions of crossed product and of polycyclic by finite group are useful for the sequel $([42], 1.5 .8,1.5 .12)$. Both of them are recalled below for the reader's convenience with a notation adapted to the present context: 
Definition 3.5. Let $\mathbf{A}$ be a ring and $\mathbf{H}$ a group. Let $\mathbf{B}$ be an over-ring of $\mathbf{A}$ and a map $\varepsilon: \mathbf{H} \rightarrow \mathbf{B}$ such that

(i) $\varepsilon$ is injective, $\varepsilon(e)=1_{\mathbf{B}}$ and all $\varepsilon(h)(h \in \mathbf{H})$ are units of $\mathbf{B}$;

(ii) for all $h, h_{1}, h_{2} \in \mathbf{H}$,

$$
\varepsilon(h) \mathbf{A}=\mathbf{A} \varepsilon(h), \varepsilon\left(h_{1} h_{2}\right) \mathbf{A}=\varepsilon\left(h_{1}\right) \varepsilon\left(h_{2}\right) \mathbf{A}=\mathbf{A} \varepsilon\left(h_{1} h_{2}\right)=\mathbf{A} \varepsilon\left(h_{1}\right) \varepsilon\left(h_{2}\right),
$$

(iii) $\mathbf{B}$ is a free right (and, by (ii), left) $\mathbf{A}$-module with basis $(\varepsilon(h))_{h \in \mathbf{H}}$.

Then $\mathbf{B}$ is called a crossed product of $\mathbf{A}$ by $\mathbf{H}$, written $\mathbf{A} * \mathbf{H}$.

Definition 3.6. Let $\mathbf{H}$ be a group with a finite chain

$$
\{e\}=\mathbf{H}_{0} \triangleleft \mathbf{H}_{1} \triangleleft \ldots \triangleleft \mathbf{H}_{n}=\mathbf{H}
$$

where $\mathbf{H}_{i} \triangleleft \mathbf{H}_{i+1}$ means that $\mathbf{H}_{i}$ is a normal subgroup of $\mathbf{H}_{i+1}$, and the factor $\mathbf{H}_{i+1} / \mathbf{H}_{i}$ is either infinite cyclic or finite. Without loss of generality, the only finite factor is $\mathbf{H}_{n} / \mathbf{H}_{n-1}$, and $\mathbf{H}$ along with the above chain is called a polycyclic by finite group (and a polycyclic group if $\mathbf{H}_{n} / \mathbf{H}_{n-1}=\{e\}$ ).

The following definition will also be needed in the sequel:

Definition 3.7. Let $\mathbf{G}$ be a Lie group; then $\mathfrak{P}^{c f}(\mathbf{G})$ is the set of all subgroups $\mathbf{H}$ of $\mathbf{G}$ such that all finitely generated subgroups of $\mathbf{H}$ are polycyclic by finite.

Note that if $\mathbf{G}$ is commutative or, more generally, if $\mathbf{G}$ is a Dedekind group, i.e. if every subgroup of $\mathbf{G}$ is normal [36], then all subgroups of $\mathbf{G}$ belong to $\mathfrak{P}^{\text {cf }}(\mathbf{G})$.

In everything that follows, we'll use the following

Notation 3.8. $\mathbf{H}$ is a subgroup of $\mathbf{G}$ and $\mathbf{A}$ is a Noetherian subalgebra of $\mathrm{U}_{\mathbf{k}}(\mathfrak{g})$.

The two main cases are $\mathbf{A}=\mathrm{U}_{\mathbf{k}}(\mathfrak{g})$-the algebra of invariant differential operators- and $\mathbf{A}=Z_{\mathbf{k}}(\mathfrak{g})$-the algebra of bi-invariant differential operators- when the latter is Noetherian $(\S$ 6.2.4). Let

$$
\mathbf{B}=\sum_{x \in \mathbf{H}} \delta_{x} \circ \mathbf{A}, \quad \mathbf{C}=\sum_{x \in \mathbf{G}} \delta_{x} \circ \mathbf{A} .
$$

Setting $\varepsilon(x) S \triangleq \delta_{x} \circ S(x \in \mathbf{G}, S \in \mathbf{A})$, this yields by (6.14) $\mathbf{B}=\mathbf{A} * \mathbf{H}$ and $\mathbf{C}=$ $\mathbf{A} * \mathbf{G}$. We have

$$
\mathbf{C}=\sum_{x \in \overline{\mathbf{G}}} \delta_{x} \circ\left(\sum_{x \in \mathbf{H}} \delta_{x} \circ \mathbf{A}\right)=\sum_{x \in \overline{\mathbf{G}}} \delta_{x} \circ \mathbf{B}
$$

where $\overline{\mathbf{G}} \subseteq \mathbf{G}$ is a set of representatives of the cosets of $\mathbf{G}$ modulo $\mathbf{H}$. All distributions belonging to $\mathbf{A}$ have their support included in $\{e\}$, thus again by (6.14) all above sums are direct since $\mathbf{G}$ is the disjoint union of its distinct cosets modulo $\mathbf{H}$. In particular, $\mathbf{B}$ is a subalgebra of $\mathbf{C}$ and

$$
\mathbf{C}=\bigoplus_{x \in \overline{\mathbf{G}}} \delta_{x} \circ \mathbf{B}=\mathbf{C} \bigotimes_{\mathbf{B}} \mathbf{B}
$$

is a free right $\mathbf{B}$-module.

When $\mathbf{G}=\mathbb{R}^{n}$ and $\mathbf{H}$ is a finitely generated subgroup of $\mathbf{G}$, then $\mathbf{B}$ is a convolution algebra of differential-difference operators with lumped shifts, denoted by $\mathbf{A}^{\text {shift }}$.

Lemma 3.9. Let $\mathbf{H} \in \mathfrak{P}^{\text {cf }}(\mathbf{G})$; then $\mathbf{B}=\mathbf{A} * \mathbf{H}$ is coherent.

Proof. Let $\mathfrak{H}$ be the directed system of all finitely generated subgroups $\mathbf{H}$ of G. The ring $\mathbf{A}$ is Noetherian, thus if $\mathbf{H}_{1} \in \mathfrak{H}$, then the ring $\mathbf{A} * \mathbf{H}_{1}$ is Noetherian again ([42], Theorem 1.5.12), thus it is coherent. Moreover, if $\mathbf{H}_{1} \subseteq \mathbf{H}_{2}$ are finitely 
generated subgroups of $\mathbf{G}$, then $\mathbf{A} * \mathbf{H}_{2}$ is a free -hence flat- left and right $\mathbf{A} * \mathbf{H}_{1}$ module ([42], Lemma 1.5.9(i)), thus

$$
\mathbf{B}=\bigcup_{\mathbf{I} \in \mathfrak{H}} \mathbf{A} * \mathbf{I}=\underset{\mathbf{I} \in \mathfrak{H}}{\lim } \mathbf{A} * \mathbf{I}
$$

is coherent according to ([5], Sect. I.2, Exercise $12(\mathrm{e}))$.

Since the two rings $\mathbf{B} \subseteq \mathbf{C}$ are subrings of $W^{\prime}, W$ is both a left and right module over $\mathbf{B}$ and over $\mathbf{C}$ via the actions $\circ$ induced by those defined in (3.2) and (3.3). The nondegenerate bilinear form $\langle-,-\rangle: W^{\prime} \times W \rightarrow \mathbf{k}$ induces a bilinear form $\langle-,-\rangle: \mathbf{C} \times W \rightarrow \mathbf{k}$. We have the following:

Lemma 3.10. The bilinear form $\langle-,-\rangle: \mathbf{C} \times W \rightarrow \mathbf{k}$ is nondegenerate.

Proof. The ring $\mathbf{C}$ contains all distributions $\delta_{x}, x \in \mathbf{G}$. If $w \in W$ is such that $\langle\mathbf{C}, w\rangle=0$, then $\left\langle\delta_{x}, w\right\rangle=w(x)=0$ for all $x \in \mathbf{G}$, thus $w=0$.

Let $R_{2} \in{ }^{q} \mathbf{B}^{k}, N=\mathbf{B}^{q} \circ R_{2}$ and $M=\mathbf{B}^{k} / \mathbf{B}^{q} \circ R_{2}$; then the sequence

$$
\mathbf{B}^{q} \stackrel{\circ R_{2}}{\longrightarrow} \mathbf{B}^{k} \longrightarrow M \longrightarrow 0
$$

is exact in ${ }_{\mathbf{B}}$ Mod and $M$ is finitely presented in that category. The map $\circ R_{2}$ is weakly* continuous (i.e. is continuous when $\mathbf{B}^{q}$ and $\mathbf{B}^{k}$ are endowed with the topologies $\sigma\left(\mathbf{B}^{q},{ }^{q} W\right)$ and $\sigma\left(\mathbf{B}^{k},{ }^{k} W\right)$ respectively) since the convolution product $\star: W^{\prime} \times W \rightarrow W$ is separately weakly continuous ([11], Chap. I, Prop. 1;2). It induces a weakly* continuous map $\circ R_{2}: \mathbf{C}^{q} \rightarrow \mathbf{C}^{k}$.

Lemma 3.11. (i) Assume that $\mathbf{H} \in \mathfrak{P}^{c f}(\mathbf{G})$. Then there exists $r \in \mathbb{N}$ and a matrix $R_{1} \in{ }^{r} \mathbf{B}^{q}$ such that the sequence below is exact in ${ }_{\mathbf{B}} \mathbf{M o d}$ :

$$
\mathbf{B}^{r} \stackrel{\circ R_{1}}{\longrightarrow} \mathbf{B}^{q} \stackrel{\circ R_{2}}{\longrightarrow} \mathbf{B}^{k}
$$

(ii) The exactness of the sequence (3.10) in ${ }_{\mathbf{B}}$ Mod is equivalent to the exactness of the sequence below in $\mathbf{C}$ Mod:

$$
\mathbf{C}^{r} \stackrel{\circ R_{1}}{\longrightarrow} \mathbf{C}^{q} \stackrel{\circ R_{2}}{\longrightarrow} \mathbf{C}^{k}
$$

Proof. (i): The ring $\mathbf{B}$ is coherent by Lemma 3.9, thus there exists a matrix $R_{1}$ such that the sequence (3.10) is exact in ${ }_{\mathbf{B}}$ Mod by Lemma 2.15(ii).

(ii): As seen above, the right $\mathbf{B}$-module $\mathbf{C}=\mathbf{C} \otimes_{\mathbf{B}} \mathbf{B}$ is free, thus faithfully flat ([39], Example (4.72)(3)), thus the exactness of the sequence (3.10) is equivalent to that of (3.11) ([39], Theorem (4.70)).

The following expresses when a fundamental principle for $W$-behaviors is valid (compare with the Ehrenpreis-Malgrange-Palamodov fundamental principle ([27], Sect. IV.2), [45], ([51], Sect. IV.5)) using the above notation:

Corollary 3.12. Let $R_{2} \in{ }^{q} \mathbf{B}^{k}$ be given and $R_{1} \in{ }^{r} \mathbf{B}^{q}$ be as in Lemma 3.11(i) if it exists. The following properties are equivalent:

(a) The fundamental principle holds, i.e. $R_{2} \circ{ }^{k} W=\left\{u \in{ }^{r} W: R_{1} \circ u=0\right\} \quad\left(R_{2} \in\right.$ $\left.{ }^{q} \mathbf{B}^{k}, R_{1} \in{ }^{r} \mathbf{B}^{q}\right)$ i.e. the equation $R_{2} \circ w=u\left(u \in{ }^{r} W\right)$ has a solution $w \in{ }^{k} W$ if, and only if $u$ satisfies the compatibility (or integrability) condition $R_{1} \circ u=0$.

(b) The map $\circ R_{2}: \mathbf{C}^{q} \rightarrow \mathbf{C}^{k}$ is strict, i.e. $\operatorname{coim}_{\mathbf{C}} \circ R_{2} \cong \operatorname{im}_{\mathbf{C}} \circ R_{2}=\mathbf{C}^{q} \circ R_{2}$.

Proof. By Lemma 3.10 and (3.4), the transpose of $R_{2} \circ:{ }^{k} W \rightarrow{ }^{q} W$ is $\circ R_{2}: \mathbf{C}^{q} \rightarrow$ $\mathbf{C}^{k}$ and that of $R_{1} \circ:{ }^{q} W \rightarrow{ }^{r} W$ is $\circ R_{1}: \mathbf{C}^{r} \rightarrow \mathbf{C}^{q}$. The fundamental principle holds 
if, and only if the sequence

$$
{ }^{k} W \stackrel{R_{2} \circ}{\longrightarrow}{ }^{q} W \stackrel{R_{1} \circ}{\longrightarrow}{ }^{r} W
$$

is exact in the category $\mathbf{V c t}_{\mathbf{k}}$ of right $\mathbf{k}$-vector spaces. By ([7], Subsect. II.6.5, Remark 1 after Corol. 4 of Prop. 7), this holds true if, and only if the map $\circ R_{2}$ : $\mathbf{C}^{q} \rightarrow \mathbf{C}^{k}$ is strict.

REMARK 3.13. (i) If $\mathbf{B}=\mathrm{U}_{\mathbf{k}}(\mathfrak{g})$, then $R_{1}$ can be constructed via the Gröbner basis algorithm which is applicable to universal enveloping algebras of finite-dimensional Lie groups ([50], Sect. 5, Definition and Corollary (37)).

(ii) Let $0 \neq \mu \in \mathcal{D}\left(\mathbb{R}^{n}\right) \subseteq W^{\prime}$, where $\mathcal{D}\left(\mathbb{R}^{n}\right)$ is the usual space of indefinitely differentiable $\mathbf{k}$-valued functions with compact support in $\mathbb{R}^{n}$; then $\mu \star W \subsetneq W$ ([44], Subsect. II.3.1, Corollary 1), thus the module ${ }_{W^{\prime}} W$ is not divisible and a fortiori not injective.

(iii) If $\mathbf{G}=\mathbb{R}^{n}$ and $\mathbf{B}=\mathbf{A}$, the fundamental principle holds ([45], Theorem 3.2), thus Condition (b) in Corollary 3.12 is always satisfied.

(iv) If $\mathbf{G}$ is solvable simply connected and $\mathbf{A}=Z_{\mathbf{k}}(\mathfrak{g})$ then ${ }_{\mathbf{A}} W$ is a divisible ([24], Prop. 2). We conjecture that $\mathbf{A} W$ is injective. Since $\mathbb{R}^{n}$ is such a group, this implies the Ehrenpreis-Malgrange-Palamodov fundamental principle, therefore if this conjecture is correct, its proof is probably difficult.

(v) If $\mathbf{A}=\mathrm{U}_{\mathbf{k}}(\mathfrak{g})$, the ring of all invariant differential operators over $\mathbf{G}$, and $\mathbf{A} W$ is divisible, then either $\mathbf{G}$ is abelian or has an abelian normal subgroup of codimension 1 ([15], Prop. 2).

(vi) If $\mathbf{G}$ is the compact abelian torus $\mathbb{R} / \mathbb{Z}$ and $\mathbf{A}=\mathrm{U}_{\mathbf{k}}(\mathfrak{g})=\mathrm{Z}_{\mathbf{k}}(\mathfrak{g})=\mathbf{k}[d / d t]$, then ${ }_{\mathbf{A}} W$ is not divisible ([62], $p$. 225), hence not injective.

(vi) If $\mathbf{G}=\mathbb{R}^{n}$ and $\mathbf{B}=\mathbf{A}^{\text {shift }}$, then ${ }_{\mathbf{B}} W$ is divisible [25].

Items (iv) and (v) of the above remark suggest preferring the commutative algebra $Z_{\mathbf{k}}(\mathfrak{g})$ of bi-invariant differential operators over the algebra $U_{\mathbf{k}}(\mathfrak{g})$ of invariant differential operators regarding the existence of global $C^{\infty}$ solutions of non-homogeneous differential equations over Lie groups.

Corollary and Definition 3.14. (1) Let $\mu$ be a left-regular element of $\mathbf{B}$. The following conditions are equivalent:

(i) $\mu \circ: W \rightarrow W$ is an epimorphism;

(ii) $\circ \mu: \mathbf{C} \rightarrow \mathbf{C}$ is a strict monomorphism, i.e. $\mathbf{C} \circ \mu \cong \mathbf{C}$.

(iii) $\circ \mu: W^{\prime} \rightarrow W^{\prime}$ is a strict monomorphism for the weak ${ }^{*}$ topology, i.e. $W^{\prime} \circ \mu \cong W^{\prime}$;

(iv) the principal ideal $W^{\prime} \circ \mu$ is weakly* closed in $W^{\prime}$;

(v) the principal ideal $W^{\prime} \circ \mu$ is strongly closed in $W^{\prime}$.

(2) A distribution $\mu \in \mathbf{B}$ is called invertible ${ }^{2}$ if it satisfies the conditions in (1).

(3) Assuming that all nonzero elements of $\mathbf{B}$ are left-regular, the left $\mathbf{B}$-module $W$ is divisible if, and only if all nonzero elements of $\mathbf{B}$ are invertible distributions, and then the B-module $W$ is faithful.

Proof. (1): Let $\mu$ be left-regular in B, i.e. $\nu \circ \mu=0$ and $\nu \in \mathbf{B}$ imply $\nu=0$ ([20], Sect. 7.1). Then the sequence (3.10) is exact with $r=0, q=k=1, R_{1}=0$ and $R_{2}=\mu$; therefore, by Lemma 3.11(ii), $\circ \mu: \mathbf{C} \rightarrow \mathbf{C}$ is a monomorphism, i.e. $\operatorname{coim}_{\mathbf{C}} \circ \mu=\mathbf{C} /$ ker $\circ \mu=\mathbf{C} /\{0\}=\mathbf{C}$. Thus, by Corollary 3.12, (i) holds if, and only if $\circ \mu: \mathbf{C} \rightarrow \mathbf{C}$ is strict, i.e. $\mathbf{C}=\operatorname{coim}_{\mathbf{C}} \circ \mu \cong \mathrm{im}_{\mathbf{C}} \circ \mu$. As a result, (i) $\Leftrightarrow($ ii).

${ }^{2}$ This denomination is in accordance with that of Ehrenpreis [26] and Hörmander ([37], Definition 16.3.12), but in a more general context. In Schwartz's terminology ([62], Sect. VI.10), such a distribution $\mu$ is called completely invertible. 
(i) $\Leftrightarrow$ (iii) by ([7], Subsect. IV.4.2, Corollary 2 of Theorem 1$)$ since $W$ is a Fréchet space, and (i) $\Leftrightarrow$ (iv) by ([7], Subsect. IV.4.2, Theorem 1).

Since $W$ is a Montel space ([13], Sect. 4, Prop. 6), it is reflexive ([7], Subsect. IV.2.5) and the weak* closure of $W^{\prime} \circ \mu$ is equal to its weak closure and therefore also to its strong closure. Therefore, (iv) $\Leftrightarrow(\mathrm{v})$.

(3): If all nonzero elements of $\mathbf{B}$ are left-regular, the divisibility of $W$ over $\mathbf{B}$ is equivalent to condition (1)(i) for all $0 \neq \mu \in \mathbf{B}$ ([39], Definition (3.16)). This condition implies the faithfulness of $W$ over $\mathbf{B}$, i.e. $\mu \circ W=0$ implies $\mu=0$.

3.3. A cogenerator property. First let us establish a general result:

Lemma 3.15. Let $\mathbf{B}=\mathbf{A} * \mathbf{H}$ be a crossed product of a Noetherian domain $\mathbf{A}$ of characteristic zero by a polycyclic by finite group $\mathbf{H}$.

(1) $\mathbf{B}$ is a semi-prime Noetherian ring, and a Noetherian domain if $\mathbf{H}$ is polycyclic.

(2) For a finitely generated left $\mathbf{B}$-module $M$, the following conditions are equivalent:

(i) $M$ is torsionless;

(ii) There exists an embedding $M \hookrightarrow \mathbf{B}^{r}$ for some $r \in \mathbb{N}$;

(iii) $M$ is torsion-free.

The same result holds for right modules.

Proof. (1): Since the ring $\mathbf{A}$ is Noetherian, so is again the ring $\mathbf{B}$ ([42], Theorem 1.5.12). In (3.8), assume without loss of generality that $\mathbf{H}_{i} / \mathbf{H}_{i-1}$ is infinite cyclic (thus isomorphic to $\mathbb{Z}$ ) for $i \leq n-1$ and finite for $i=n$. (a) The ring $\mathbf{A} * \mathbb{Z}$ is isomorphic to a skew Laurent polynomial ring $\mathbf{A}\left[X, X^{-1} ; \alpha\right]$ where $\alpha$ is an automorphism of $\mathbf{A}$ ([42], Proposition 1.5.11), thus it is an integral domain of characteristic zero. Thus for $i \leq n-1, \mathbf{A} * \mathbf{H}_{i} \cong\left(\mathbf{A} * \mathbf{H}_{i-1}\right) * \mathbb{Z}$ is an integral domain of characteristic zero by induction. (b) We have $\mathbf{B} \cong\left(\mathbf{A} * \mathbf{H}_{n-1}\right) *\left(\mathbf{H}_{n} / \mathbf{H}_{n-1}\right)$ where $\mathbf{H}_{n} / \mathbf{H}_{n-1}$ is finite, thus $\mathbf{B}$ is semiprime by ([42], Theorem 10.5.11).

(2): Since (1) holds true, Conditions (i), (ii) and (iii) are equivalent by ([42], Prop. 3.4.3 and 3.4.7).

Let $\mathbf{G}$ be a Lie group and consider $W$-behaviors $(W=\mathcal{E}(\mathbf{G}))$ over the ring $\mathbf{B}=$ $\mathbf{A} * \mathbf{H}$ where $\mathbf{H}$ is a polycyclic by finite subgroup of $\mathbf{G}$. Thus let $\mathbf{C}=\mathbf{A} * \mathbf{G}$, $R_{1} \in{ }^{q} \mathbf{B}^{k}, N=\mathbf{B}^{q} \circ R_{1} \subseteq \mathbf{B}^{k}, M=\mathbf{B}^{k} / N, \mathfrak{B}=\mathfrak{B}_{W}(M)=N^{\perp}$ and $\mathfrak{B}^{\perp}=N^{\perp \perp}=$ $\left\{T \in \mathbf{B}^{k}: T \circ \mathfrak{B}\right\}=0$.

THEOREM 3.16. (1) If the finitely presented module $M$ satisfies the equivalent conditions of Part (2) of Lemma 3.15, then the following equivalent properties hold:

(i) The canonical map $\phi_{M}: M \rightarrow W^{\mathfrak{B}}: m \mapsto(m \circ w)_{w \in \mathcal{B}}$ is injective;

(ii) $N=N^{\perp \perp}$.

(2) $W$ is a cogenerator for the full subcategory $\mathcal{D}$ of ${ }_{\mathbf{B}} \mathbf{M o d}^{f p}$, all objects of which are torsionless (or equivalently torsion-free).

Proof. (1): By Lemma 2.15(iv) and Lemma 3.11(ii), there exists a matrix $R_{2} \in$ ${ }^{k} \mathbf{B}^{r}$ such that the sequences (3.10), (3.11) are exact. In particular, with $N=\mathbf{B}^{q} \circ R_{1}$, the module $\mathbf{C} \bigotimes_{\mathbf{B}} N=\mathbf{C}^{q} \circ R_{1}=\operatorname{ker}_{\mathbf{C}} \circ R_{2}$ is closed, hence by Lemma 3.10 and Theorem 2.23(i), $\mathbf{C} \bigotimes_{\mathbf{B}} N=\left(\mathbf{C} \bigotimes_{\mathbf{B}} N\right)^{\perp \perp}$ and the canonical map

$$
\tilde{\phi}_{\tilde{M}}: \tilde{M} \rightarrow W^{\mathfrak{B}}: \tilde{m} \mapsto(\tilde{m} \circ w)_{w \in \mathfrak{B}}
$$

$\left(\tilde{M}=\mathbf{C} \otimes_{\mathbf{B}} M, \tilde{m}=1 \otimes m\right)$ is injective. By (3.9), the map

$$
\Psi: M \rightarrow \tilde{M}: m \mapsto 1 \otimes m=\delta_{e} \otimes m
$$

is injective too. In addition, $\bullet \phi_{M}=\bullet \Psi \tilde{\phi}_{\tilde{M}}$ since $m \circ w=(1 \otimes m) \circ w$ for the given identification, hence $\bullet \phi_{M}$ is injective. 
(2): This is a straightforward consequence of (1)(i) and of Corollary and Definition 2.8 .

REMARK 3.17. (i) If $M \in{ }_{\mathbf{B}}$ Mod $^{f p}$ is not torsion-free, $W$ is not a cogenerator of the category consisting of $M$ alone, as shown by ([31], Example 2.3).

(ii) All B-modules $M \in \mathcal{D}$ are Hausdorff by Lemma 3.15(2)(ii) since the map $\circ R_{2}$ in (3.10) induces a continuous injection $M=\mathbf{B}^{k} / \mathbf{B}^{q} R_{1} \hookrightarrow \mathbf{B}^{r}$ where $\mathbf{B}^{r} \subseteq\left(W^{\prime}\right)^{r}$ is Hausdorff.

(iii) Theorem 3.16 is new, even in the standard case of delay-differential systems with $\mathbf{G}=\mathbb{R}$ and $\mathbf{H}=\bigoplus_{1 \leq i \leq m} \mathbb{Z} \tau_{i}$ with delays $\tau_{1}, \ldots, \tau_{m}$ (thus $\mathbf{B}=\mathbf{k}\left[d / d t, \sigma, \sigma^{-1}\right]$, $\left.\sigma=\left(\sigma_{1}, \ldots, \sigma_{m}\right), \sigma^{-1} \triangleq\left(\sigma_{1}^{-1}, \ldots, \sigma_{m}^{-1}\right), \sigma_{i}: w(t) \mapsto w\left(t+\tau_{i}\right)\right) . \quad$ Nevertheless, Theorem 3.16 has connections with the results in ([72], Lemmas 3.5 and 3.7) which are slightly generalized below:

Lemma 3.18. (Compare with [72], Lemmas 3.5 and 3.7). Let $\mathbf{B}$ be a ring and $W$ a left $\mathbf{B}$-module with a free element $w$, i.e. such that the map $\varphi_{w}: \mathbf{B} \rightarrow W: b \mapsto b w$ is injective. Then $W$ is a cogenerator for the full subcategory $\mathcal{D}$ of $\mathbf{B} \mathbf{M o d}$, all objects of which are torsionless $\mathbf{B}$-modules. The category $\mathcal{D}$ contains all modules $M$ for which there exists an embedding $M \hookrightarrow \mathbf{B}^{r}, r \geq 0$, and such modules are described in Lemma 2.15(iv).

If $\mathbf{B}$ is a domain, the assumption means that the module $W$ is not a torsion module.

Proof. If $M$ is torsionless, there exists by definition an embedding $\psi: M \hookrightarrow \mathbf{B}^{I}$ for some set of indices $I$ (see $\S \mathbf{2 . 2 . 2}$ ). The assumption implies that

$$
\varphi_{w}^{I}: \mathbf{B}^{I} \rightarrow W^{I}:\left(b_{i}\right)_{i \in I} \mapsto\left(b_{i} w\right)_{i \in I}
$$

is again a monomorphism, and so is the composition

$$
\phi_{M}: M \stackrel{\psi}{\longrightarrow} \mathbf{B}^{I} \stackrel{\varphi_{w}^{I}}{\longrightarrow} W^{I},
$$

therefore $W$ is has the desired cogenerator property according to Corollary and Definition 2.8. $\mathrm{c}$

When the Lie group $\mathbf{G}$ is solvable, Lemma 3.18 implies Theorem 3.16, as shown by the following:

Lemma 3.19. Assuming that the Lie group $\mathbf{G}$ is solvable, the left $\mathrm{U}_{\mathbf{k}}(\mathfrak{g})$-module $W$ is not torsion, and neither is the $\mathbf{A}$-module $W$.

Proof. (a) Consider the sequence (6.15), let $\mathfrak{a}$ be a one-codimensional ideal of $\mathfrak{g}$ and $\mathbf{k} X=\mathfrak{g} / \mathfrak{a}$, thus $\mathfrak{g}=\mathfrak{a} \oplus \mathbf{k} X$. The restriction $\delta$ to $\mathrm{U}_{\mathbf{k}}(\mathfrak{a})$ of the adjoint map $\operatorname{ad}(X)$, i.e. $\delta: \mathrm{U}_{\mathbf{k}}(\mathfrak{a}) \rightarrow \mathrm{U}_{\mathbf{k}}(\mathfrak{a}): r \mapsto r X-X r$, is an inner derivation. By the Poincaré-Birkhoff-Witt theorem (Appendix 2, §6.2.4), $\mathrm{U}_{\mathbf{k}}(\mathfrak{g})=\bigoplus_{n>0} \mathrm{U}_{\mathbf{k}}(\mathfrak{a}) X^{n}$, thus $\mathrm{U}_{\mathbf{k}}(\mathfrak{g})=\mathrm{U}_{\mathbf{k}}(\mathfrak{a})[X ; \delta]$ is a skew polynomial ring with indeterminate $X$ over $\mathrm{U}_{\mathbf{k}}(\mathfrak{a})$.

(b) Let $\mathbf{R}$ be a k-algebra, $\delta: \mathbf{R} \rightarrow \mathbf{R}$ a k-derivation, $\mathbf{S}=\mathbf{R}[X ; \delta]$, and let $\mathbf{R}^{*}=$ $\operatorname{Hom}_{\mathbf{k}}(\mathbf{R}, \mathbf{k}), \mathbf{S}^{*}=\operatorname{Hom}_{\mathbf{k}}(\mathbf{S}, \mathbf{k})$ be the duals of $\mathbf{R}$ and $\mathbf{S}$, respectively. Generalizing ([50], Sect. 3, Lemma (29)), we obtain the following:

If the (left or right) $\mathbf{R}$-module $\mathbf{R}^{*}$ is not a torsion module, then neither is the (left or right) $\mathbf{R}$-module $\mathbf{S}^{*}$.

This is obvious when $\delta=0$ since then

$$
\mathbf{S}^{*}=\operatorname{Hom}_{\mathbf{k}}(\mathbf{S}, \mathbf{k}) \cong \operatorname{Hom}_{\mathbf{k}}\left(\mathbf{R}^{(\mathbb{N})}, \mathbf{k}\right) \cong{ }^{\mathbb{N}} \operatorname{Hom}_{\mathbf{k}}(\mathbf{R}, \mathbf{k})={ }^{\mathbb{N}} \mathbf{R}^{*}
$$

(Appendix 1, §6.1.2), and if the $\mathbf{R}$-module ${ }^{\mathbb{N}} \mathbf{R}^{*}$ is torsion, then $\mathbf{R}^{*}$ is torsion too; this rationale can be extended to the general case but we skip the technical details due to space limitation. 
(c) By induction, we obtain from the above that $\mathrm{U}_{\mathbf{k}}(\mathfrak{g})^{*}$ is not a torsion (left or right) $\mathrm{U}_{\mathbf{k}}(\mathfrak{g})$-module, and the same holds for $W$ due to the Borel epimorphism (Appendix 2, §6.2.4).

(d) Since $\mathbf{A} \subseteq \mathrm{U}_{\mathbf{k}}(\mathfrak{g}), W$ is not a torsion A-module.

3.4. Dense image representation. Let us study torsion-free and torsionless controllability as in Subsect. 2.2.

Theorem 3.20. Let $(\mathbf{B}, \circ)$ be a convolution algebra, $R_{1} \in{ }^{q} \mathbf{B}^{k}, N=\mathbf{B}^{q} \circ R_{1}$, $M=\mathbf{B}^{k} / N$, and $\mathfrak{B}=\mathfrak{B}_{W}(M)=\left\{w \in{ }^{k} W: R_{1} \circ w=0\right\}$.

(1) Assume that $\mathbf{G}$ is a Lie group and that $\mathbf{H} \in \mathfrak{P}^{c f}(\mathbf{G})$, so that $\mathbf{B}=\mathbf{A} * \mathbf{H}$ is coherent according to Lemma 3.9. If $M$ is torsionless, then it is Hausdorff and $\mathfrak{B}$ admits a dense image representation, i.e. the following property $(\boldsymbol{P})$ holds:

$(\boldsymbol{P})$ : There are $r \in \mathbb{N}$ and $R_{2} \in{ }^{k} \mathbf{B}^{r}$ such that $\mathfrak{B}=\overline{\left.R_{2} \circ{ }^{r} W\right)}$ (where the closure is taken in the usual Fréchet topology of compact convergence in $\left.{ }^{r} W\right)$.

In addition, if $\mathbf{H}$ is polycyclic by finite, then $M$ is torsionless if, and only if it is torsion-free by Lemma 3.15.

(2) Conversely, if $\mathbf{B}$ is dense in $W^{\prime}, M$ is Hausdorff and $\mathfrak{B}$ admits a dense image representation, then $M$ is both torsion-free and torsionless.

(3) If $\mathbf{B}$ is an Ore domain (e.g. when $\mathbf{G}$ is a Lie group and $\mathbf{B}=\mathbf{A} * \mathbf{H}$ where $\mathbf{H}$ is polycyclic by Lemma 3.15(1)) and $\langle-,-\rangle: \mathbf{B} \times W \rightarrow \mathbf{k}$ is nondegenerate, or in other words if $\mathbf{B}$ is wealkly* dense in $W^{\prime}$, then $M$ is torsion-free if, and only if $M$ is Hausdorff (i.e. $N$ is closed in $\mathbf{B}^{k}$ ) and $\mathfrak{B}$ admits a dense image representation.

Proof. (1) By Lemma 2.15(iv) there exists a matrix $R_{2} \in{ }^{k} \mathbf{B}^{r}$ such that both sequences (3.10), (3.11) are exact. The exactness of (3.10) implies that $N=\operatorname{im}_{\mathbf{B}}\left(\circ R_{1}\right)=\operatorname{ker}_{\mathbf{B}}\left(\circ R_{2}\right)$. Since $\circ R_{2}: \mathbf{B}^{k} \rightarrow \mathbf{B}^{r}$ is continuous and $\mathbf{B}$ is Hausdorff, the kernel $N$ of this map is closed and the factor $M=\mathbf{B}^{k} / N$ is Hausdorff. The exactness of (3.11) implies that $\operatorname{im}_{\mathbf{C}}\left(\circ R_{1}\right)=\operatorname{ker}_{\mathbf{C}}\left(\circ R_{2}\right)$. Therefore, by $([7]$, Subsect. II.6.3, Corol. 3 of Prop. 4) and ([7], Subsect. II.6.4, Corol. 4 of Prop. 6),

$$
\overline{R_{2}{ }^{r} W}=\left(\operatorname{ker}_{\mathbf{C}} \circ R_{2}\right)^{0}=\left(\operatorname{im}_{\mathbf{C}} \circ R_{1}\right)^{0}=\operatorname{ker}_{W}\left(R_{1} \circ\right)=\mathfrak{B}
$$

where the closure is taken in the topology $\sigma\left({ }^{r} W, \mathbf{C}^{r}\right)$ or equivalently in the initial Fréchet topology of ${ }^{r} W$.

(2) Since $M=\mathbf{B}^{k} / N$ is Hausdorff, $N=\mathbf{B}^{q} \circ R_{1}$ is closed in $\mathbf{B}^{k}$. In addition, the bilinear form $\langle-,-\rangle: \mathbf{B} \times W \rightarrow \mathbf{k}$ is nondegenerate since $\mathbf{B}$ is dense -thus weakly* dense- in $W^{\prime}$ ([7], Subsect. II.6.2, Corol. 4 of Prop. 3). Therefore, $N=N^{\perp \perp}=\mathfrak{B}^{\perp}$ by $2.23(\mathrm{i})$, thus $N=\left(\overline{R_{2}{ }^{r} W}\right)^{\perp}=\left(R_{2} \circ{ }^{r} W\right)^{\perp}=\operatorname{ker}_{\mathbf{B}} \circ R_{2}$. Thus the sequence (3.10) is exact and $M$ is both torsion-free and torsionless by Lemma 2.15(iii).

(3) (a) Necessary condition: Since $\mathbf{B}$ is an Ore domain, the finitely generated torsion-free module $M$ admits by Lemma 2.15(iv) a continuous embedding in some $\mathbf{B}^{r}$ and there exists an exact sequence (3.10). Since the form $\langle-,-\rangle: \mathbf{B} \times W \rightarrow \mathbf{k}$ is nondegenerate, the property $\overline{R_{2}{ }^{r}{ }^{r} W}=\mathfrak{B}$ is shown as in (1).

(b) The proof of the sufficient condition is the same as that of (2).

Corollary 3.21. Assume $\mathbf{G}=\mathbb{R}^{n}$ with the data of Theorem 3.20. Then $W^{\prime}$ is a commutative domain and so is any convolution algebra $\mathbf{B} . \quad A$ module $M=\mathbf{B}^{k} / \mathbf{B}^{q} \circ R_{1}$ is torsion-free if, and only if it admits an embedding in some $\mathbf{B}^{r}$. Therefore, Theorem 3.20 gets the following form:

(1) If $\mathbf{H}$ is any subgroup of $\mathbb{R}^{n}, \mathbf{B}=\mathbf{A} * \mathbf{H}$ and $M$ is torsion-free, then $N=\mathbf{B}^{q} \circ R_{1}$ is closed in $\mathbf{B}^{k}$, and $\mathfrak{B}$ admits a dense image representation.

(2) If $\langle-,-\rangle: \mathbf{B} \times W \rightarrow \mathbf{k}$ is nondegenerate, i.e. if $\mathbf{B}$ is wealkly* dense in $W^{\prime}$, 
then $M$ is torsion-free if, and only if $M$ is Hausdorff and $\mathfrak{B}$ admits a dense image representation.

REMARK 3.22. (i) It can be deduced from ([67], Theorems 11 and 14) that when $\mathbf{G}=\mathbb{R}, \mathbf{B}=W^{\prime}$ and $M$ is Hausdorff, then $\mathfrak{B}$ admits a dense image representation if, and only if $M$ is torsion-free. Corollary 3.21 is both more precise and more general. (ii) The matrix $R_{2}$ in Theorem 3.20(1) can be constructed using the Gröbner basis algorithm.

\section{Differential systems with distributed shifts over Lie groups.}

4.1. The ring $\mathcal{H}(\mathbf{B})$ and its basic properties. Recall that $\mathbf{A}$ is a Noetherian subalgebra of $\mathbf{U}_{\mathbf{k}}(\mathfrak{g})$ (Notations 3.1 and 3.8). In addition, in the sequel we make the following

ASSUMPTion 4.1. $\mathbf{H}$ is a polycyclic subgroup of $\mathbf{G}$ such that all nonzero elements of $\mathbf{B}=\mathbf{A} * \mathbf{H}$ are invertible distributions (Corollary and Definition 3.14).

Example 4.2. (i) If $\mathbf{G}=\mathbb{R}^{n}$, Assumption 4.1 is satisfied if $\mathbf{A} \triangleq \mathbf{U}_{\mathbf{k}}(\mathfrak{g})=$ $\mathbf{k}\left[\frac{\partial}{\partial x_{1}}, \ldots, \frac{\partial}{\partial x_{n}}\right]$ and $\mathbf{H}$ is any finitely generated subgroup of $\mathbf{G}$ by Remark 3.13(vi).

(ii) Let $\mathbf{G}$ be a solvable simply connected Lie group such that $\mathbf{A} \triangleq \mathbf{Z}_{\mathbf{k}}(\mathfrak{g})$ is Noetherian (not all solvable Lie group have this property: see § 6.2.4). Then Assumption 4.1 is satisfied with $\mathbf{H}=\{$ e $\}$ by Remark 3.13(iv).

The ring $\mathcal{H}(\mathbf{B})$ studied below is a generalization of the ring $\mathcal{H}$ introduced, in the case $\mathbf{G}=\mathbb{R}$, by Gluesing-Luerssen [31] when the delays are commensurable (see also [32]) and by Habets [35] without this assumption. By Lemma 3.15(1), B is a Noetherian domain, thus an Ore domain, and it admits a quotient division ring $\mathrm{Q}(\mathbf{B})$ of left fractions $(a)^{\circ-1} \circ b$ and of right fractions $b \circ(a)^{\circ-1}$, where $c=(a)^{\circ-1} \circ b$ (resp. $c=b \circ(a)^{\circ-1}$ ) means by definition that $a \circ c=b$ (resp. $c \circ a=b$ ).

Recall the following: (a) $\mu \circ \nu=\mu \star \nu$ for any $\mu, \nu \in W^{\prime}$ by Lemma 3.2(ii). (b) A ring $\mathbf{R}$ is called weakly finite if for any integer $n \geq 1$, every generating set of $n$ elements in $\mathbf{R}^{n}$ is free; over for such a ring, a square matrix is left- (or right-) invertible if, and only if it is invertible ([18], Sect. 0.2).

Theorem and Definition 4.3. (1) Let

$$
\mathcal{H}(\mathbf{B}) \triangleq\left\{b \circ(a)^{\circ-1} \in \mathrm{Q}(\mathbf{B}): \exists \mu \in W^{\prime}, b=\mu \circ a\right\} .
$$

Then $\mathcal{H}(\mathbf{B})$ is a subring of both $\mathrm{Q}(\mathbf{B})$ and $W^{\prime}$, and $\mathcal{H}(\mathbf{B})=\mathrm{Q}(\mathbf{B}) \cap W^{\prime}$.

(2) The following conditions are equivalent for a right fraction and $b \circ(a)^{\circ-1} \in \mathrm{Q}(\mathbf{B})$ :

(i) $b \circ(a)^{\circ-1} \in \mathcal{H}(\mathbf{B})$;

(ii) $\operatorname{ker}_{W} a \circ \subseteq \operatorname{ker}_{W} b \circ$

(3) $\mathcal{H}(\mathbf{B})$ is an Ore domain, thus is weakly finite, and its quotient division ring is $\mathrm{Q}(\mathbf{B})$.

(4) When $\mathbf{G}=\mathbb{R}$ and $\mathbf{H} \neq\{e\}$ is cyclic, $\mathcal{H}(\mathbf{B})$ is not Noetherian.

Proof. (1) is clear. (2) By definition, $b \circ(a)^{\circ-1} \in \mathrm{Q}(\mathbf{B})$ belongs to $\mathcal{H}(\mathbf{B})$ if, and only if there exists $\mu \in W^{\prime}$ such that $b=\mu \circ a$. Let $w \in \operatorname{ker}_{W} a \circ$; then $a \circ w=0$ and $b \circ w=(\mu \circ a) \circ w=\mu \circ(a \circ w)=0$ by Lemma 3.2(iii), which implies that $\operatorname{ker}_{W} a \circ \subseteq \operatorname{ker}_{W} b \circ$.

Conversely, let $a, b \in \mathbf{B}, a \neq 0$, such that $\operatorname{ker}_{W} a \circ \subseteq \operatorname{ker}_{W} b$.

For any $\mu \in \mathbf{B}$, let $\mathfrak{B}_{\mu}=\left(W^{\prime} \circ \mu\right)^{\perp}$. We have $\mathfrak{B}_{\mu}=\operatorname{ker}_{W} \mu \circ$ and $\overline{W^{\prime} \circ \mu}=\mathfrak{B}_{\mu}^{\perp}$ by Theorem 2.23(i) since $\langle-,-\rangle: W^{\prime} \times W \rightarrow \mathbf{k}$ is nondegenerate. By Corollary and Definition 3.14(iii), $\overline{W^{\prime} \circ \mu}=W^{\prime} \circ \mu$. 
Thus the assumption $\operatorname{ker}_{W} a \circ \subseteq \operatorname{ker}_{W} b \circ$ implies $W^{\prime} \circ b \subseteq W^{\prime} \circ a$, hence $b \in W^{\prime} \circ a$ and $b \circ(a)^{\circ-1} \in \mathcal{H}(\mathbf{B})$.

(3) $\mathcal{H}(\mathbf{B})$ is an integral domain since $\mathcal{H}(\mathbf{B}) \subseteq \mathrm{Q}(\mathbf{B})$.

Let us show that $\mathcal{H}(\mathbf{B})$ is right Ore, i.e. whenever $f_{1}, f_{2} \in \mathcal{H}(\mathbf{B})^{\times}, f_{1} \circ \mathcal{H}(\mathbf{B}) \cap$ $f_{2} \circ \mathcal{H}(\mathbf{B}) \neq 0$, in other words there exist $g_{1}, g_{2} \in \mathcal{H}(\mathbf{B})^{\times}$such that $f_{1} \circ g_{1}=f_{2} \circ g_{2}$. This happens if, and only if $g_{2}=\left(f_{2}^{\circ-1} \circ f_{1}\right) \circ g_{1}$ where $f_{2}^{\circ-1} \circ f_{1} \in \mathrm{Q}(\mathbf{B})$. There exist $b, a \in \mathbf{B}^{\times} \subseteq W^{\prime \times}$ such that $f_{2}^{\circ-1} \circ f_{1}=b \circ(a)^{\circ-1}$. Take $g_{1}=a, g_{2}=b$. Then $g_{1}, g_{2} \in \mathcal{H}(\mathbf{B})^{\times}$and $f_{1} \circ g_{1}=f_{2} \circ g_{2}$ as desired.

The definition of $\mathcal{H}(\mathbf{B})$ is left/right symmetric, thus $\mathcal{H}(\mathbf{B})$ is a left Ore domain too.

An Ore domain is weakly finite according to ([18], Sect. 0.8, Corollary 8.7).

We have $\mathbf{B} \subseteq \mathcal{H}(\mathbf{B}) \subseteq \mathrm{Q}(\mathbf{B})$, thus the quotient division ring of $\mathcal{H}(\mathbf{B})$ is $\mathrm{Q}(\mathbf{B})$.

(4) When $\mathbf{G}=\mathbb{R}$ and $\mathbf{H} \neq\{e\}$ is cyclic, then $\mathcal{H}(\mathbf{B})$ is not Noetherian by ([31], Prop. 3.1(c)).

Remark 4.4. (a) Part (1) of Theorem and Definition 4.3 generalizes ([32], §3.5) where its assumed that $\mathbf{G}=\mathbb{R}$ and $\mathbf{H} \neq\{e\}$ is cyclic (see also [10]).

(b) In addition, Corollary and Definition 3.14(3) implies that Assumptions 2.1 and 4.1 of Habets [35] are satisfied, thus $\mathcal{H}(\mathbf{B})$ is the over-ring of $\mathbf{B}$ denoted by $\mathbf{B}_{W}$ in ([35], Definition 3.1); therefore, extending Habets' terminology to the noncommutative case, $\mathcal{H}(\mathbf{B})$ is the ring of all admissible right fractions $b \circ(a)^{\circ-1} \in \mathrm{Q}(\mathbf{B})$ with respect to $W$.

4.2. Cogenerator properties. Since $\mathcal{H}(\mathbf{B}) \subseteq W^{\prime}, W$ has a canonical structure of $\mathcal{H}(\mathbf{B})$-module, by restriction of the ring of scalars.

Theorem 4.5. For $i=1,2$, let $R_{i} \in{ }^{q_{i}} \mathcal{H}(\mathbf{B})^{k}, U_{i}=W^{\prime q_{i}} \circ R_{i}$ and $\mathfrak{B}_{i}=U_{i}^{\perp}=$ $\operatorname{ker}_{W}\left(R_{i} \circ\right)$. Assume that $\mathrm{rk} R_{2}=q_{2}$. Then:

(i) The map $R_{2} \circ:{ }^{k} W \rightarrow{ }^{q_{2}} W$ is surjective; in particular all nonzero distributions belonging to $\mathcal{H}(\mathbf{B})$ are invertible and the $\mathcal{H}(\mathbf{B})$-module $W$ is both divisible and faithful. (ii) $\mathfrak{B}_{2}^{\perp}=U_{2}^{\perp \perp}=U_{2}$ where $\mathfrak{B}_{2}^{\perp}=\mathfrak{B}_{2}^{\perp\left(W^{\prime}\right)} \triangleq\left\{w^{\prime} \in{ }^{k} W^{\prime}: w^{\prime} \circ \mathfrak{B}_{2}\right\}=0$.

(iii) $\operatorname{ker}_{W}\left(R_{2} \circ\right) \subseteq \operatorname{ker}_{W}\left(R_{1} \circ\right)$ if, and only if there exists $X \in{ }^{q_{i}} \mathcal{H}(\mathbf{B})^{q_{2}}$ such that $R_{1}=X \circ R_{2}$.

(iv) The $\mathcal{H}(\mathbf{B})$-module $W$ is a cogenerator for the full subcategory $\mathcal{D}$ of $\mathcal{H}(\mathbf{B}) \mathbf{M o d}$, the objects $M$ of which are finitely presented by a short exact sequence

$$
0 \longrightarrow \mathcal{H}(\mathbf{B})^{q} \stackrel{\circ R}{\longrightarrow} \mathcal{H}(\mathbf{B})^{k} \longrightarrow M \longrightarrow 0 .
$$

Proof. (i): The assumption means that $R_{2}$ is right-invertible over $\mathrm{Q}(\mathbf{B})$ and implies that $R_{2}$ is right-invertible over $\mathrm{Q}(\mathbf{B})$, i.e. there exists a matrix $X_{2} \in{ }^{k} \mathrm{Q}(\mathbf{B})^{q_{2}}$ such that $R_{2} \circ X_{2}=I_{q_{2}}$. Since $\mathbf{B}$ is an Ore domain, for each $j \in\left\{1, \ldots, q_{2}\right\}$, all entries of the $j$-th column of $X_{2}$ have a common right denominator $\mu_{j} \in \mathbf{B}^{\times}([42]$, Prop. 2.1.16(i)), i.e. there exist elements $\left(Y_{2}\right)_{i j} \in \mathbf{B}(1 \leq i \leq k)$ such that $\left(X_{2}\right)_{i j}=$ $\left(Y_{2}\right)_{i j} \circ \mu_{j}^{\circ-1}$; then $\left(Y_{2}\right)_{i j}=\left(X_{2}\right)_{i j} \circ \mu_{j} \in \mathbf{B}$ and

$$
R_{2} \circ Y_{2}=R_{2} \circ\left(X_{2} \circ \operatorname{diag}\left(\mu_{j}\right)_{1 \leq j \leq q_{2}}\right)=\operatorname{diag}\left(\mu_{j}\right)_{1 \leq j \leq q_{2}} .
$$

Therefore, for any $w \in{ }^{q_{2}} W, R_{2} \circ\left(Y_{2} \circ w\right)=\operatorname{diag}\left(\mu_{j}\right)_{1 \leq j \leq q_{2}} \circ w=\left(\mu_{j} \circ w_{j}\right)_{1 \leq j \leq q_{2}}$. By hypothesis, each distribution $\mu_{j}$ is invertible and $\mu_{j} \circ: W \rightarrow W$ is surjective. Therefore, $R_{2} \circ:{ }^{k} W \rightarrow{ }^{q_{2}} W$ is surjective too. 
(ii): The transpose of $R_{2} \circ:{ }^{k} W \rightarrow{ }^{q_{2}} W$ is $\circ R_{2}: W^{\prime q_{2}} \rightarrow W^{\prime k}$ and the spaces ${ }^{k} W$, $q_{2} W$ are Fréchet spaces. Therefore, by (i) and ([37], Lemma 16.5.8), $\circ R_{2}: W^{\prime q_{2}} \rightarrow$ $W^{\prime k}$ is injective and $W^{\prime q_{2}} \circ R_{2}$ is weakly* closed in $W^{\prime k}$. Therefore, $\mathfrak{B}_{2}^{\perp}=U_{2}^{\perp \perp}=U_{2}$ by Theorem $2.23(\mathrm{i})$ since $\langle-,-\rangle:{ }^{k} W^{\prime} \times W^{k} \rightarrow \mathbf{k}$ is nondegenerate.

(iii): The sufficient condition is obvious. Let us prove the necessary condition. If $\mathfrak{B}_{2} \subseteq \mathfrak{B}_{1}$, then $W^{\prime q_{1}} \circ R_{1} \subseteq \mathfrak{B}_{1}^{\perp} \subseteq \mathfrak{B}_{2}^{\perp}$ and $\mathfrak{B}_{2}^{\perp}=W^{\prime q_{2}} \circ R_{2}$ by (ii). Thus $W^{\prime q_{1}} \circ R_{1} \subseteq W^{\prime q_{2}} \circ R_{2}$, hence every row of $R_{1}$ and therefore $R_{1}$ itself are left-multiples of $R_{2}$, i.e. there exists $X \in{ }^{q_{2}} W^{\prime q_{1}}$ such that $R_{1}=X \circ R_{2}$. Since rk $R_{2}=q_{2}$, after a possible permutation of columns we obtain

$$
R_{i}=\left[\begin{array}{ll}
R_{i}^{\prime} & R_{i}^{\prime \prime}
\end{array}\right] \in^{q_{i}} \mathcal{H}(\mathbf{B})^{q_{i}+\left(k-q_{i}\right)} \quad(i=1,2)
$$

where $R_{2}^{\prime}$ is invertible over $\mathrm{Q}(\mathbf{B})$. Then $R_{1}^{\prime}=X \circ R_{2}^{\prime}$ and $X=R_{1}^{\prime} \circ R_{2}^{\prime \circ-1}$. As in (i) we obtain $Y \in{ }^{q_{1}} \mathbf{B}^{q_{2}}$ and $\nu_{i} \in \mathbf{B}^{\times}\left(1 \leq i \leq q_{2}\right)$ with $R_{2}^{\prime} \circ Y=\operatorname{diag}\left(\nu_{i}\right)_{1 \leq i \leq q_{2}}=\Delta$, hence

$$
X=R_{1}^{\prime} \circ R_{2}^{\prime \circ-1}=R_{1}^{\prime} \circ Y \circ \Delta^{\circ-1} \in{ }^{q_{1}}\left(\mathrm{Q}(\mathbf{B}) \bigcap W^{\prime}\right)^{q_{2}}={ }^{q_{1}} \mathcal{H}(\mathbf{B})^{q_{2}} .
$$

(iv) is a consequence of (iii) and of Theorem 2.13(iii). $\mathrm{Z}$

Corollary 4.6. Let $R \in{ }^{q} \mathcal{H}(\mathbf{B})^{k}, N=\mathcal{H}(\mathbf{B})^{q} \circ R, M=\mathcal{H}(\mathbf{B})^{k} / N, \mathfrak{B}=$ $N^{\perp}=\operatorname{ker}_{W}(R \circ)$.

(i) $\mathrm{rk} R=q$ if, and only if $R \circ{ }^{k} W={ }^{q} W$.

(ii) If $R$ has a left-inverse $X$ with coefficients in $W^{\prime}$, i.e. $X \in{ }^{k} W^{\prime q}, X \circ R=\delta_{e} I_{k}$, then $\mathfrak{B}=0$.

(iii) Assuming that $\mathrm{rk} R=q, \operatorname{ker}_{W}(R \circ)=0$ if and only if $R \in \mathrm{GL}_{q}(\mathcal{H}(\mathbf{B}))$.

(iv) In particular, for $R \in \mathcal{H}(\mathbf{B})$, $\operatorname{ker}_{W}(R \circ)=0$ if, and only if $R$ is a unit in $\mathcal{H}(\mathbf{B})$.

Proof. (i): The necessary condition follows from Theorem 4.5(i).

Conversely, assume rk $R<q$. Since rk $R$ is (by definition) the dimension of the left $\mathrm{Q}(\mathbf{B})$-vector space generated by the rows of $R$, there exists a nonzero row $y \in \mathrm{Q}(\mathbf{B})^{q}$ such that $y \circ R=0$. Let $d$ be a common left denominator of all elements of $y$ and $x=d \circ y \in \mathcal{H}(\mathbf{B})^{q}$; then $x \circ R=0$ and $x \neq 0$. Thus $R \circ\left({ }^{k} W\right) \subseteq \operatorname{ker}_{W}(x \circ) \subseteq{ }^{q} W$. Assuming that $\operatorname{ker}_{W}(x \circ)={ }^{q} W$, then $x \circ{ }^{q} W=0$ and $x_{i} \circ W=0$ for all $i \in\{1, \ldots, q\}$, a contradiction since the $\mathcal{H}(\mathbf{B})$-module $W$ is faithful by Theorem $4.5(\mathrm{i})$. Therefore $R \circ\left({ }^{k} W\right) \subsetneq{ }^{q} W$.

(iii): Assume that $r k R=q$. Clearly, $\operatorname{ker}_{W}(R \circ)=0$ if, and only if $\operatorname{ker}_{W}(R \circ) \subseteq$ $\operatorname{ker}_{W}\left(I_{k} \delta_{e} \mathrm{O}\right)$, and by Theorem 4.5 (iii) this is equivalent to the existence of a matrix $X \in{ }^{k} \mathcal{H}(\mathbf{B})^{q_{2}}$ such that $I_{k} \delta_{e}=X \circ R$, thus $R$ is left-invertible over $\mathcal{H}(\mathbf{B})$ and rk $R=k$. Therefore, $R$ is square, and since $\mathcal{H}(\mathbf{B})$ is weakly finite by Theorem and Definition $4.3(2)$, the left-invertibility of the square matrix $R$ over $\mathcal{H}(\mathbf{B})$ is equivalent to its invertibility over that ring.

(ii) is obvious and (iv) is a particular case of (iii).

REMARK 4.7. Consider the data of Theorem 4.5 and Corollary 4.6 for the special case $\mathbf{G}=\mathbb{R}$.

(a) Statement (iii) of Theorem 4.5 is proved in ([33], Theorem 5.3) without any assumption on $\mathrm{rk} R_{2}$ when $\mathbf{H} \neq 0$ is cyclic. In that case, indeed, $\mathcal{H}(\mathbf{B})$ is a Bézout domain, thus $R_{2}$ is left-equivalent to a matrix $\left[\begin{array}{c}Q \\ 0\end{array}\right]$ where $Q$ is full row rank ([18],

Sect. 1.1, Theorem 1.1(g)), thus the assumption on $\mathrm{rk} R_{2}$ becomes superfluous. Except in that case, the statement of Theorem 4.5 is not valid when the matrix $R$ is not assumed to be full row rank, as shown by ([66], Example after the proof of Theorem 
3).

(b) Statement (iii) of Theorem 4.5 can be viewed as an extension to the noncommutative case of ([35], Theorem 4.9) (see Remark 4.4(b)).

(c) The units in $\mathcal{H}(\mathbf{B})$ are explicitly described in Corollary 4.12 below when $\mathbf{G}=\mathbb{R}^{n}$.

4.3. Differential systems with distributed shifts over $\mathbb{R}^{n}$. In what follows, $\mathbf{G}=\mathbb{R}^{n}$ and $\mathbf{H}$ is a finitely generated subgroup of $\mathbf{G}$. The ring of all entire complexvalued functions over $\mathbb{C}^{n}$ is denoted by $\mathcal{O}\left(\mathbb{C}^{n}\right)$. For $s, z \in \mathbb{C}^{n}$, we set

$$
s \bullet z \triangleq \sum_{1 \leq i \leq n} s_{i} z_{i}, \quad|s|=\left(\sum_{1 \leq i \leq n}\left|s_{i}\right|^{2}\right)^{1 / 2} .
$$

Let $\mathbf{A} \triangleq \mathrm{U}_{\mathbf{k}}(\mathfrak{g})=\mathbf{k}\left[\frac{\partial}{\partial x_{1}}, \ldots, \frac{\partial}{\partial x_{n}}\right]$ and $\mathbf{B}=\mathbf{A} * \mathbf{H} . \quad$ The convolution algebra $\mathbf{B}$ is now a commutative domain, thus the left fractions $a^{\circ-1} \circ b \in \mathrm{Q}(\mathbf{B})$ coincide with the right fractions $b \circ a^{\circ-1} \in \mathrm{Q}(\mathbf{B})$ and are denoted by $b /{ }^{\circ} a$ in the sequel. As is well known, the Laplace transform

$$
\mathcal{L}: W^{\prime} \ni \mu \mapsto \hat{\mu}(s) \triangleq \int_{\mathbb{R}^{n}} e^{-s \bullet x} d \mu(x), \quad s \in \mathbb{C}^{n}
$$

yields the following isomorphisms:

$$
\mathbf{A} \cong \mathbb{C}[s], \quad \mathbf{B} \cong \mathcal{P} \mathcal{E}(\mathbf{H}) \triangleq \sum_{\tau \in \mathbf{H}} \mathbb{C}[s] e^{-\tau \bullet s}, \quad W^{\prime} \cong P W\left(\mathbb{C}^{n}\right)
$$

where $P W\left(\mathbb{C}^{n}\right)$ is the set of all functions $f \in \mathcal{O}\left(\mathbb{C}^{n}\right)$ for which there exist $c>0$, $a>0$ and $N \in \mathbb{N}$ such that

$$
|f(s)| \leq c(1+|s|)^{N} e^{a|\operatorname{Re}(s)|} .
$$

The elements of $\mathcal{P E}(\mathbf{B})$ are called complex exponential-polynomial functions [2] and the third isomorphism is given by the Paley-Wiener-Schwartz theorem ([22], (22.18.7)).

The following results complement Corollary and Definition 3.14 and are due to Ehrenpreis and Malgrange; they are nicely expounded by Hörmander [37] and are gathered below for convenience:

Lemma 4.8. ([37], Theorems 16.3.10, 16.5.7 and 16.5.19; [1], Appendix). For $\mu \in W^{\prime}$, consider the convolution equation

$$
\mu \star u=f
$$

where the distribution $\mu$ is given and the distribution $u$ is looked for. The following conditions are equivalent:

(i) If $f \in W^{\prime}$ and $\hat{f}(s) / \hat{\mu}(s) \in \mathcal{O}\left(\mathbb{C}^{n}\right)$, then there exists a (unique) $u \in W^{\prime}$ such that $\hat{f}(s) / \hat{\mu}(s)=\hat{u}(s)$ (which implies (4.1));

(ii) Equation (4.1) has a solution $u \in W$ for any $f \in W$, i.e. $\mu$ is invertible;

(iii) Equation (4.1) has a solution $u \in \mathcal{D}^{\prime}\left(\mathbb{R}^{n}\right)$ for any $f \in \mathcal{D}^{\prime}\left(\mathbb{R}^{n}\right)$

(iv) $\hat{\mu}$ is slowly decreasing ([37], Theorem 16.3.10 and Definition 16.3.12; [1]).

Remark 4.9. Malgrange established the equivalence between Corollary and Definition 3.14(1)(v) and Lemma 4.8(i) for $0 \neq \mu \in \mathcal{E}^{\prime}\left(\mathbb{R}^{n}\right)$ ([44], Corollary on p. 310). Ehrenpreis [26] introduced the notion of slowly decreasing $\hat{\mu}$ and proved the equivalences (ii) $\Leftrightarrow($ iii $) \Leftrightarrow$ (iv) of Lemma 4.8. The elements of $\mathcal{P} \mathcal{E}(\mathbf{H})$ are easily seen to be slowly decreasing, and this proves Ehrenpreis' result in Remark 3.13(vi). 
The following was proved and partially stated by Berenstein and Dostal [2] when $n \geq 1$, and is explicit in [35] when $n=1$ :

Lemma 4.10. Let $F, G \in \mathcal{P E}(\mathbf{H}), G \neq 0$, be such that $F / G \in \mathcal{O}\left(\mathbb{C}^{n}\right)$. There exist $H \in \mathcal{P E}(\mathbf{H})$ and $0 \neq P \in \mathbb{C}[s]$ such that $F / G=H / P$.

Proof. (a) By ([2], Main Theorem), there exist $H \in \mathcal{P E}\left(\mathbb{R}^{n}\right)$ and $0 \neq P \in \mathbb{C}[s]$ such that $F / G=H / P$.

(b) For any $0 \neq L(s) \in \mathcal{P} \mathcal{E}\left(\mathbb{R}^{n}\right)$, write $L(s)=\sum_{\tau \in \mathbf{T}} l_{\tau}(s) e^{-\tau \bullet s}$ where $\mathbf{T}$ is a finite subset of $\mathbb{R}^{n}$ and $0 \neq l_{\tau}(s) \in \mathbb{C}[s]$ for any $\tau \in \mathbf{T}$. The elements $\tau \in \mathbf{T}$ are the exponents [35] (or, more loosely speaking, the "frequencies" [2]) of $L(s)$. The above element $H \in \mathcal{P E}\left(\mathbb{R}^{n}\right)$ is constructed by induction in ([2], Proof of Theorem 1). At each step, the exponents involved are $\mathbb{Z}$-linear combinations of those of $F$ and $G$, i.e. belong to $\mathbf{H}$; see ([2], (2.20)-(2.26)) for more details. Since the procedure stops in a finite number of steps, $H \in \mathcal{P E}(\mathbf{H})$. (When $n=1$, the fact that the exponents of $H$ are $\mathbb{Z}$-linear combinations of those of $F$ and $G$ is used in the proof of ([35], Theorem 5.9).)

The following is a generalization of ([35], Lemma 3.2 and Theorems 5.4, 5.7 and 5.9) to the case $n \geq 1$ :

THEOREM 4.11. The following rings coincide with the ring $\mathcal{H}(\mathbf{B})$ :

$$
\begin{aligned}
& \mathcal{H}_{1}(\mathbf{B})=\left\{b /{ }^{\circ} a \in \mathrm{Q}(\mathbf{B}): \hat{b}(s) / \hat{a}(s) \in P W\left(\mathbb{C}^{n}\right)\right\}, \\
& \mathcal{H}_{2}(\mathbf{B})=\left\{b /{ }^{\circ} a \in \mathrm{Q}(\mathbf{B}): \hat{b}(s) / \hat{a}(s) \in \mathcal{O}\left(\mathbb{C}^{n}\right)\right\}, \\
& \mathcal{H}_{3}(\mathbf{B})=\left\{b /{ }^{\circ} a \in \mathrm{Q}(\mathbf{B}) \cap W^{\prime}: b \in \mathbf{B}, a \in \mathbf{A}^{\times}\right\} .
\end{aligned}
$$

Proof. (a) By definition, if $u=b /{ }^{\circ} a \in \mathcal{H}(\mathbf{B})$, then $\hat{u}(s) \in P W\left(\mathbb{C}^{n}\right) \subseteq \mathcal{O}\left(\mathbb{C}^{n}\right)$ which proves that $\mathcal{H}(\mathbf{B}) \subseteq \mathcal{H}_{1}(\mathbf{B})$. The inclusion $\mathcal{H}_{1}(\mathbf{B}) \subseteq \mathcal{H}_{2}(\mathbf{B})$ is obvious.

(b) Let $b \in \mathbf{B}, a \in \mathbf{B}^{\times}$be such that $\hat{b}(s) / \hat{a}(s) \in \mathcal{O}\left(\mathbb{C}^{n}\right)$. By Lemma 4.8, the distribution $a$ is invertible and there exists $u \in W^{\prime}$ such that $a \circ u=b$. Therefore, $b /{ }^{\circ} a \in \mathcal{H}(\mathbf{B})$, hence $\mathcal{H}_{2}(\mathbf{B}) \subseteq \mathcal{H}(\mathbf{B})$.

(c) Last, $\mathcal{H}_{3}(\mathbf{B})=\mathcal{H}_{2}(\mathbf{B})$ by Lemma 4.10 .

Corollary 4.12. An element $T \in \mathcal{H}(\mathbf{B})$ is a unit if, and only if it has the form $T=\alpha \delta_{\tau}, \tau \in \mathbf{H}, \alpha \in \mathbf{k}^{\times}$, i.e., is also a unit in $\mathbf{B}$.

Proof. The indicated elements are obviously units. The ring $\mathbf{B}$ can be written $\mathbf{A}\left[\sigma, \sigma^{-1}\right]$ with the notation in Remark 3.17(iii) and $\mathbf{A}=\mathbf{k}[D], D=\left(\partial / \partial x_{i}\right)_{1 \leq i \leq n}$. Assume that $T_{1} \in \mathcal{H}(\mathbf{B})$ is invertible and that

$$
\delta_{0}=T_{1} \circ T_{2}, T_{i}=b_{i} /{ }^{\circ} a_{i}, b_{i} \in \mathbf{B}, a_{i} \in \mathbf{A}^{\times}(i=1,2)
$$

therefore $b_{1} \circ b_{2}=a_{1} \circ a_{2}$ in $\mathbf{B}=\mathbf{A}\left[\sigma, \sigma^{-1}\right]$, where we have used $\mathcal{H}(\mathbf{B})=\mathcal{H}_{3}(\mathbf{B})$. We infer $b_{1}=a_{1}^{\prime} \circ \delta_{\tau_{1}}$ for some $a_{1}^{\prime} \in \mathbf{A}$ and $\tau_{1} \in \mathbf{H}$, thus $T_{1}=b_{1} /{ }^{\circ} a_{1}=\left(a_{1}^{\prime} /{ }^{\circ} a_{1}\right) \circ \delta_{\tau_{1}}$. Since $\hat{T}_{1}(s)$ is entire, so is the rational function $\hat{a}_{1}^{\prime}(s) / \hat{a}_{1}(s)=\hat{a}_{1}^{\prime \prime}(s)$. Thus $\hat{a}_{1}^{\prime \prime}(s)$ is a polynomial and $T_{1}=a_{1}^{\prime \prime} \circ \delta_{\tau_{1}}$ with $a_{1}^{\prime \prime} \in \mathbf{A}$. The same argument applies to $T_{2}$ and furnishes

$$
\delta_{0}=T_{1} \circ T_{2}=\left(a_{1}^{\prime \prime} \circ a_{2}^{\prime \prime}\right) \circ \delta_{\tau_{1}+\tau_{2}}
$$

thus $a_{1}^{\prime \prime} \circ a_{2}^{\prime \prime}=\delta_{0}$ and $\tau_{1}+\tau_{2}=0$. Since $\mathbf{A}$ is a polynomial algebra, $a_{1}^{\prime \prime}=\alpha \in \mathbf{k}^{\times}$, hence $T=\alpha \delta_{\tau}$ as asserted. 
REMARK 4.13. (a) We consider the space $\mathcal{P E} \triangleq \mathcal{P E}\left(\mathbb{R}^{n}\right)$ of real polynomialexponential functions $\left(\mathcal{P} \mathcal{E} \subseteq \mathcal{E}\left(\mathbb{R}^{n}\right)\right)$. As proved by Gurevič ([34], Theorem 3.2), there exists a column $R \in{ }^{k} \overline{\mathcal{E}}^{\prime}\left(\mathbb{R}^{n}\right), n \geq 2, k=6$, such that

$$
0=\operatorname{ker}_{\mathcal{P E}}(R \circ)={ }^{k}(\mathcal{P E}) \bigcap \operatorname{ker}_{W}(R \circ), \quad \operatorname{ker}_{W}(R \circ) \neq 0
$$

i.e. $R \circ w=0$ has nonzero solutions in $W$, but no polynomial-exponential ones. Thus $\operatorname{ker}_{\mathcal{P E}}(R \circ)=\operatorname{ker}_{\mathcal{P E}}(1 \circ)=0$ and according to Malgrange's theorem ([44], Preliminaries, Sect. 4, Theorem 3), there exists a row $X \in \mathcal{O}\left(\mathbb{C}^{n}\right)^{k}$ such that $X \hat{R}=1$. However, $\operatorname{ker}_{P W\left(\mathbb{C}^{n}\right)}(\hat{R} \bullet) \nsubseteq \operatorname{ker}_{P W\left(\mathbb{C}^{n}\right)}(1 \bullet)=0$, so that $([66]$, Theorem 3) does not hold true for $n>1$. This is because this result is based on Schwartz-Kelleher-Taylor's theorem on mean periodic vector-valued functions ([38], Theorem 7.3) (see also [52]) which implies that for any natural integer $k$, every shift-invariant closed subspace of ${ }^{k} W$ is spanned by the ${ }^{k} \mathbb{C}$-valued exponential-polynomial functions it contains when $n=1$. Gurevič's counter-example shows that the last result does not hold for $n>1$.

(b) With the notation from Remark 3.17(iii), the ring $\mathcal{H}(\mathbf{B})=\mathcal{H}_{3}(\mathbf{B})$ is isomorphic to $\mathbf{D} \cap \mathcal{O}\left(\mathbb{C}^{n}\right)$ where $\mathbf{D}=\mathbf{k}(s)[\sigma], s=\left(s_{1}, \ldots s_{n}\right), \sigma=\left(\sigma_{1}, \ldots, \sigma_{m}\right)$. The ring $\mathbf{D}$ is projective-free ([40], Sect. IV.4, Corol. 4.10), thus a finitely presented D-module $M$ has a full row rank matrix of definition if, and only $M$ has projective dimension $\leq 1$. Assume $m>1$. Since $\mathbf{D}$ has global dimension $m$ ([42], 7.5.3(iv)), such a module $M$ is very peculiar, and so are probably also the finitely presented $\mathcal{H}(\mathbf{B})$-modules in Theorem 4.5(iv), for we conjecture that $\mathcal{H}(\mathbf{B})$ has global dimension $\geq m$.

5. Concluding remarks. Using the definition of a cogenerator for a subcategory, many results of the literature have been generalized: see, e.g., Theorems 2.10, $2.13,2.23,3.16$ and 4.5 . Surprisingly, the statement of several results is purely algebraic whereas their proof is based on the theory of locally convex topological spaces in duality: see, e.g., Theorems 3.16 and 4.5. Section 3 can be extended to systems over general locally compact groups using Bruhat's theory [13], with the same proofs. A natural extension of this paper is the study of the fundamental principle and of the cogenerator properties for analytic differential-difference systems; this is the subject of a forthcoming paper [9].

\section{Appendices.}

6.1. Appendix 1: Preabelian and semi-abelian categories. We refer to MacLane's classical book [41] for a general presentation of categories and functors. A preabelian category is an additive category in which every morphism has a kernel and a cokernel, thus an image and a coimage ([57], Sect. 2.1). All categories considered in this paper are preabelian, except when otherwise stated.

6.1.1. Objects and morphisms. Consider a category $\mathcal{C}$, its class of objects $\mathrm{Ob}(\mathcal{C})$ and its class of morphisms $\operatorname{Mor}(\mathcal{C})$. We write $X \in \mathcal{C}($ instead of $X \in \mathrm{Ob}(\mathcal{C}))$ to mean that $X$ is an object of $\mathcal{C}$. The opposite of $\mathcal{C}$, denoted by $\mathcal{C}^{\text {op }}$, is deduced from $\mathcal{C}$ by "reversing the arrows". This leads to define left and right morphisms. To clarify this, consider the category Set such that Ob (Set) is the class of all sets and $\operatorname{Hom}_{\text {Set }}(X, Y)$ is the set of all maps $f: X \rightarrow Y$. If $f$ is written $x \mapsto x f$ (resp. $x \mapsto f x$ ), we call it a left (resp. right) map since $f$ acts on the left (resp. on the right), and to avoid confusions $f$ can be written $\bullet f$ (resp. $f \bullet$ ). Consider the composition

$$
X \stackrel{f}{\longrightarrow} Y \stackrel{g}{\longrightarrow} Z .
$$


If $f$ and $g$ are left (resp. right) maps, this composition is $f g$ (resp. $g f$ ). Now, let $\mathcal{C}$ be any category and consider the composition (6.1) where $f, g \in \operatorname{Mor}(\mathcal{C})$. Generalizing the above, we'll say that $f$ and $g$ are left morphisms (written $\bullet f$ and $\bullet g$ to avoid confusions) if this composition is denoted as $f g$, and right morphisms (written $f \bullet$ and $g \bullet$ ) if this composition is denoted as $g f$. For example, in [41], all morphisms are right ones whereas in ([20], Chap. 2), all morphisms are left ones. In the rest of this appendix, all morphisms are right ones.

All facts below are classical. An isomorphism is a bimorphism, i.e. both a monomorphism and an epimorphism, but the converse does not hold in general. Let $X$ be an object of $\mathcal{C}$. The set $P_{X}$ of all morphisms with target $X$ has preorder $\leqq$ with $u \bullet \leqq v \bullet$ to mean that $u$ factors through $v$ (i.e. $u=v u^{\prime}$ for some morphism $u^{\prime} \bullet$ ). Two morphisms $u, v \in P_{X}$ are called equivalent if both $u \bullet \leqq v \bullet$ and $v \bullet \leqq u \bullet$. Dually, the set $Q^{X}$ of all morphisms with source $X$ has preorder $\geqq$ with $f \bullet \geqq g \bullet$ to mean that $g$ factors through $f$ (i.e. $g=g^{\prime} f$ for some $g^{\prime} \bullet$ ) and $f, g$ are called equivalent if both $f \bullet \geqq g \bullet$ and $g \bullet \geqq f \bullet$. A subobject of $X$ is an equivalence class of monomorphisms belonging to $P_{X}$. Thus a representative of a subobject of $X$ is a pair $(Y, \iota)$ such that $\iota: Y \rightarrow X$ is a monomorphism, called the canonical injection (or the inclusion) $Y \hookrightarrow X$. Dually, a factor object of $X$ is an equivalence class of epimorphisms belonging to $Q^{X}$. A representative of a factor of $X$ is a pair $(Z, \varphi)$ such that $\varphi: X \rightarrow Z$ is an epimorphism, called the canonical epimorphism $X \rightarrow Z$. Abusing the language, $(Y, \iota)$ is denoted by $\iota$ or $Y$, and $(Z, \varphi)$ by $\varphi$ or $Z$, when there is no risk of confusion. The above applies to kernels, cokernels, images and coimages. Let $f \bullet: X \rightarrow Y$; then it is convenient to think of ker $f$ as the largest subobject $(K, \kappa \bullet)$ of $X$ annihilated by $f$ (i.e. such that $f \kappa=0$ ), and of coker $f$ as the largest factor $(C, \gamma \bullet)$ of $Y$ annihilating $f$ (i.e. such that $\gamma f=0)$. The image and the coimage of $f$, respectively written $\operatorname{im} f$ and $\operatorname{coim} f$, are defined as

$$
\operatorname{im} f=\operatorname{ker} \operatorname{coker} f, \quad \operatorname{coim} f=\operatorname{coker} \operatorname{ker} f ;
$$

they are respectively a subobject of $Y$ and a factor of $X$. Let $u \in P_{X}$ and $g \in Q^{X}$. Then $u \leqq \operatorname{ker} g \Leftrightarrow g u=0 \Leftrightarrow$ coker $u \geqq g$, which means that the two functions ker : $Q^{X} \rightarrow P_{X}$, coker : $P_{X} \rightarrow Q^{X}$ constitute an order-reversing Galois connection. In particular,

$$
\begin{aligned}
\operatorname{ker} g & =\text { ker coker ker } g=\operatorname{ker} \operatorname{coim} g=\operatorname{im} \operatorname{ker} g, \\
\operatorname{coker} u & =\operatorname{coker} \operatorname{ker} \operatorname{coker} u=\operatorname{coker} \operatorname{im} u=\operatorname{coim} \operatorname{coker} u .
\end{aligned}
$$

Therefore: (i) $\iota$ is a kernel if, and only if $\iota=\operatorname{im} \iota$, (ii) $\beta$ is a cokernel if, and only if $\beta=\operatorname{coim} \beta$, (iii) if $\iota=\operatorname{im} f$, then $\operatorname{coker} \iota=\operatorname{coker} f$ and $\operatorname{im} \iota=\operatorname{im} f=\iota$.

Let $f: X \rightarrow Y$. There exists a unique morphism $f_{\text {ind }}$, called the induced morphism, such that $f$ admits the factorization

$$
f: X \stackrel{\alpha}{\rightarrow} \operatorname{coim} f \stackrel{f_{\text {ind }}}{\longrightarrow} \operatorname{im} f \stackrel{\omega}{\hookrightarrow} Y
$$

where $\alpha$ is the canonical epimorphism and $\omega$ is the inclusion. The morphism $f$ is called strict if $f_{\text {ind }}$ is an isomorphism. A preabelian category $\mathcal{C}$ is called semi-abelian if for any morphism $f$, the induced morphism $f_{\text {ind }}$ is a bimorphism ([60], p. 168); if for each morphism $f, f_{\text {ind }}$ is an isomorphism, then $\mathcal{C}$ is called abelian.

6.1.2. Functors. All categories are semi-abelian in what follows, and all functors are additive in this paper. Recall that a functor $\mathfrak{F}: \mathcal{C} \rightarrow \mathcal{D}$ is said to be (i) full 
(resp. faithful, fully faithful) if its arrow-map is surjective (resp. injective, bijective), (ii) injective (resp. surjective, bijective) if its object map has this property, (iii) an isomorphism if it is fully faithful and bijective. The image of $\mathfrak{F}$, written $\mathfrak{F}(\mathcal{C})$, is a class consisting of two subclasses: that of all objects $\mathfrak{F}(X), X \in \mathcal{C}$, and that of all morphisms $\mathfrak{F}(f), f \in \operatorname{Mor}(\mathcal{C})$. The image $\mathfrak{F}(\mathcal{C})$ is a subcategory of $\mathcal{D}$ if $\mathfrak{F}$ is injective ([47], Sect. II.10). A sequence

$$
X_{1} \stackrel{f_{1}}{\longrightarrow} X_{2} \stackrel{f_{2}}{\longrightarrow} X_{3} \longrightarrow 0
$$

is called exact if $f_{2}$ (or, more specifically, $\left(f_{2}, X_{3}\right)$ ) is a cokernel of $f_{1}$. Dually, a sequence

$$
X_{1} \stackrel{f_{1}}{\longleftarrow} X_{2} \stackrel{f_{2}}{\longleftarrow} X_{3} \longleftarrow 0
$$

is called exact if $f_{2}$ (or, more specifically, $\left(f_{2}, X_{3}\right)$ ) is a kernel of $f_{1}$. A covariant functor $\mathfrak{F}: \mathcal{C} \rightarrow \mathcal{D}$ is left exact if exactness of (6.7) implies exactness of

$$
\mathfrak{F}\left(X_{1}\right) \stackrel{\mathfrak{F}\left(f_{1}\right)}{\longleftarrow} \mathfrak{F}\left(X_{2}\right) \stackrel{\mathfrak{F}\left(f_{2}\right)}{\longleftarrow} \mathfrak{F}\left(X_{3}\right) \longleftarrow 0 .
$$

Dually, a contravariant functor $\mathfrak{F}: \mathcal{C} \rightarrow \mathcal{D}$ is left exact if it is a left exact covariant functor $\mathcal{C}^{\mathrm{op}} \rightarrow \mathcal{D}$. The bifunctor $\operatorname{Hom}_{\mathcal{C}}(\bullet, \bullet): \mathcal{C} \times \mathcal{C} \rightarrow \mathbf{A b}$ (where $\mathbf{A b}$ denotes the category of abelian groups and $\mathbb{Z}$-linear maps) is left exact in each argument, covariant in the first one and contravariant in the second one. An object $X$ is called projective in $\mathcal{C}$ if $\operatorname{Hom}_{\mathcal{C}}(X, \bullet)$ transforms epimorphisms into surjections, and injective in $\mathcal{C}$ if it is projective in $\mathcal{C}^{\mathrm{op}}$. Assuming that $\mathcal{C}$ has products and coproducts indexed by the set $I$ (possibly filtering),

$\operatorname{Hom}_{\mathcal{C}}\left(\coprod_{i \in I} X_{i}, Y\right) \cong \prod_{i \in I} \operatorname{Hom}_{\mathcal{C}}\left(X_{i}, Y\right), \operatorname{Hom}_{\mathcal{C}}\left(X, \prod_{i \in I} Y_{i}\right) \cong \prod_{i \in I} \operatorname{Hom}_{\mathcal{C}}\left(X, Y_{i}\right)$

where the isomorphisms are canonical, thus are identifications. A coproduct in $\mathcal{C}$ is a product in $\mathcal{C}^{\mathrm{op}}$. We define the power (resp. the copower) $U^{I}=\prod_{i \in I} U_{i}$ (resp. $\left.U^{(I)}=\coprod_{i \in I} U_{i}\right), U_{i}=U$. An object $U$ of category $\mathcal{C}$ with arbitrary coproducts is a generator in $\mathcal{C}$ if the following equivalent conditions are satisfied: (i) every object of $\mathcal{C}$ is isomorphic to a factor of a copower $U^{(I)}$; (ii) for any nonzero right morphism $f \bullet: X \rightarrow Y$, there exists $\alpha \bullet: U \rightarrow X$ such that $f \alpha \neq 0$; (iii) the functor $\operatorname{Hom}_{\mathcal{C}}(U, \bullet)$ is faithful. An object $W$ of a category $\mathcal{C}$ with arbitrary products is called a cogenerator in $\mathcal{C}$ if it is a generator in $\mathcal{C}^{\text {op }}$.

6.2. Appendix 2: Lie groups and Lie algebras. We refer to Bourbaki's and Dieudonné's treatises [8], [22] for an exhaustive presentation of Lie groups and Lie algebras; the convolution of distributions and the differential operators over Lie groups are expounded in the latter.

6.2.1. Lie groups. All Lie groups are real and locally compact in this paper. A Lie group $\mathbf{G}$ is a real analytic manifold which is a group (multiplicatively noted in general, with neutral element $e$, and additively noted if $\mathbf{G}$ is abelian, with neutral element 0) such that the map $\mathbf{G} \times \mathbf{G} \ni(x, y) \mapsto x^{-1} y \in \mathbf{G}$ is analytic. Let $a \in$ $\mathbf{G}$; there exists a chart $(V, \xi)$ such that $V$ is an open connected neighborhood of $a$, $\xi: V \rightarrow \Omega$ is a diffeomorphism onto an open connected neighborhood $\Omega$ of 0 in $\mathbb{R}^{n}$ (where $n$ is the dimension of the manifold $\mathbf{G}$ ) and $\xi(a)=0$; such a chart is said to be centered at $a$. Let $f: V \rightarrow \mathbf{k}$ (where $\mathbf{k}$ is the field of real or complex numbers) 
be a $C^{\infty}$ function. There exists a uniquely defined $C^{\infty}$ function $\phi: \Omega \rightarrow \mathbf{k}$ such that $f(x)=\phi\left(\xi_{1}(x), \ldots, \xi_{n}(x)\right)$ where the analytic functions $\xi_{i}$ are the local coordinates (which can also be considered as variables). For any multi-index $\alpha=\left(\alpha_{1}, \ldots, \alpha_{n}\right) \in \mathbb{N}^{n}$ and any $x \in V$, we set ([12], Part I, Sect. 2.2)

$$
|\alpha|=\sum_{1 \leq i \leq n} \alpha_{i}, \quad \alpha !=\prod_{1 \leq i \leq n} \alpha_{i} ! \quad \frac{\partial^{\alpha}}{\partial \xi^{\alpha}}=\frac{\partial^{|\alpha|}}{\partial \xi_{1}^{\alpha_{1}} \ldots \partial \xi_{n}^{\alpha_{n}}}, \quad D_{\xi}^{\alpha} f(x)=\frac{\partial^{\alpha} \phi}{\partial \xi^{\alpha}}(\xi(x)) .
$$

6.2.2. Distributions with compact support. Let $\mathcal{E}(\mathbf{G})$ be the space of all $C^{\infty}$ functions $\mathbf{G} \rightarrow \mathbf{k}$, endowed with its usual Fréchet topology of compact convergence ([22], (17.3.1)). The space $\mathcal{E}^{\prime}(\mathbf{G})$ of all continuous linear forms $\mathcal{E}(\mathbf{G}) \rightarrow \mathbf{k}$ is the space of distributions with compact support over $\mathbf{G}$. For $T \in \mathcal{E}^{\prime}(\mathbf{G})$ and $\varphi \in \mathcal{E}(\mathbf{G}), T(\varphi)$ is written

$$
\langle T, \varphi\rangle=\langle\varphi, T\rangle^{\sim}
$$

or, with a slight abuse of notation ([22], (17.3.8))

$$
\int_{\mathbf{G}} d T(x) \varphi(x)=\int_{\mathbf{G}} \varphi(x) d T(x) .
$$

Let $(V, \xi)$ be a chart centered at $a \in \mathbf{G}$; the restriction $\left.T\right|_{V}$ is the linear form $\varphi \mapsto$ $T(\varphi), \varphi \in \mathcal{E}(V)$, and its derivative with multi-index $\alpha$ is expressed in function of $(V, \xi)$ by $\left\langle D_{\xi}^{\alpha} T, \varphi\right\rangle=(-1)^{|\alpha|}\left\langle T, D_{\xi}^{\alpha} \varphi\right\rangle$.

Let $T, S \in \mathcal{E}^{\prime}(\mathbf{G})$; their convolution product $T \star S \in \mathcal{E}^{\prime}(\mathbf{G})$ is the linear form $\mathcal{E}(\mathbf{G}) \ni \varphi \mapsto\langle T \otimes S, \varphi m\rangle \in \mathbf{k}$ where $m$ is the map $(x, y) \mapsto x y$. By (6.10), this can also be written $([22],(14.5 .4),(17.10 .3))$

$$
\int_{\mathbf{G}} \varphi d(T \star S)=\int_{\mathbf{G}}\left(\int_{\mathbf{G}} \varphi(x y) d T(x)\right) d S(y)=\int_{\mathbf{G}} d S(y) \int_{\mathbf{G}} d T(x) \varphi(x y) .
$$

Let $a \in \mathbf{G}$ and $\delta_{a}: \varphi \mapsto \varphi(a)$ be the Dirac distribution at point $a$. From the above,

$$
\begin{aligned}
D_{\xi}^{\alpha} \varphi(a) & =(-1)^{|\alpha|}\left\langle D_{\xi}^{\alpha} \delta_{a}, \varphi\right\rangle, \\
\left\langle\delta_{a} \star S, \varphi\right\rangle & =\langle S, \varphi(a \bullet)\rangle, \quad\left\langle T \star \delta_{a}, \varphi\right\rangle=\langle T, \varphi(\bullet a)\rangle
\end{aligned}
$$

where $\varphi(a \bullet)$ and $\varphi(\bullet a)$ respectively denote the functions $x \mapsto \varphi(a x)$ and $x \mapsto \varphi(x a)$.

The convolution algebra $\left(\mathcal{E}^{\prime}(\mathbf{G}), \star\right)$ is associative with unit element $\delta_{e}$, and is commutative if, and only if $\mathbf{G}$ is commutative too. This ring is an integral domain in the classical case $\mathbf{G}=\mathbb{R}^{n}$ according to the Paley-Wiener-Schwartz theorem ([22], 22.18.7), but not in general. For example, let $\mathbb{T}$ be the torus, $C$ a nonzero constant; then $0 \neq C \in \mathcal{E}^{\prime}(\mathbb{T}), \dot{\delta}_{0} \neq 0$ and $\dot{\delta}_{0} \star C=0$.

Let $d x$ be a left Haar measure and for any function $f \in \mathcal{E}(\mathbf{G})$ consider the distribution $T=f d x$. The support of $T$ is not compact in general but for any $S \in$ $\mathcal{E}^{\prime}(\mathbf{G})$, the convolution products $T \star S$ and $S \star T$ exist and are absolutely continuous with respect to $d x$, with density function $g$ (resp. $h$ ) belonging to $\mathcal{E}(\mathbf{G})$. Identifying $f \star S$ with $g$ and $S \star f$ with $h$ one obtains ([22], (14.8.2), (14.8.4))

$$
(S \star f)(a)=\int_{\mathbf{G}} f\left(s^{-1} a\right) d S(s),(f \star S)(a)=\int_{\mathbf{G}} f\left(a s^{-1}\right) \Delta\left(s^{-1}\right) d S(s)
$$


and in particular

$$
\delta_{a} \star f=f\left(a^{-1} \bullet\right), \quad f \star \delta_{a}=\Delta\left(a^{-1}\right) f\left(\bullet a^{-1}\right)
$$

where $\Delta$ is the modular function which is positive ([22], Sect. XIV.3) and analytic ([8], Subsect. III.3.16, Prop. 56). The operator $f(\bullet) \mapsto f(a \bullet)($ resp. $f(\bullet) \mapsto f(\bullet a)$ is the left (resp. right) shift denoted by $\gamma\left(a^{-1}\right)$ (resp. $\left.\boldsymbol{\delta}(a)\right)([22],(14.1 .1)$. Let $a \in \mathbf{G},(V, \xi)$ a chart centered at $e$, and $f \in \mathcal{E}(\mathbf{G})$; as easily shown, for any $x \in a V$,

$$
D_{\xi}^{\alpha} \delta_{a} \star f(x)=D_{\xi}^{\alpha} f\left(a^{-1} x\right)
$$

Denoting by $\mathcal{T}_{x}^{\infty}(\mathbf{G})$ the $\mathbf{k}$-vector space consisting of all $\mathbf{k}$-valued distributions with support included in $\{x\}(x \in \mathbf{G})$ and by $\mathcal{T}^{\infty}(\mathbf{G})$ the $\mathbf{k}$-vector space consisting of all punctual $\mathbf{k}$-valued distributions (i.e. all $\mathbf{k}$-valued distributions with finite support), $\mathcal{T}_{e}^{\infty}(\mathbf{G})$ and $\mathcal{T}^{\infty}(\mathbf{G})$ are subalgebras of $\left(\mathcal{E}^{\prime}(\mathbf{G}), \star\right)$, and $\mathcal{T}^{\infty}(\mathbf{G})$ is commutative if, and only if $\mathbf{G}$ is commutative too. In addition,

$$
\mathcal{T}^{\infty}(\mathbf{G})=\bigoplus_{x \in \mathbf{G}} \mathcal{T}_{x}^{\infty}(\mathbf{G})
$$

and $\mathcal{T}_{x}^{\infty}(\mathbf{G})=\delta_{x} \star \mathcal{T}_{e}^{\infty}(\mathbf{G})=\mathcal{T}_{e}^{\infty}(\mathbf{G}) \star \delta_{x}([8]$, Subsect. III.3.1).

6.2.3. Lie algebra of a Lie group. Let $\mathfrak{g} \triangleq T_{e}(\mathbf{G})$ be the tangent space of $\mathbf{G}$ at $e$. Consider the $n$ first order elementary differential operators

$$
X_{i}=\left.\partial_{i}\right|_{e}: f \rightarrow D_{\xi}^{\alpha} f(e), \quad \alpha_{j}=\delta_{i j} \quad i, j \in\{1, \ldots, n\}
$$

(where $\delta_{i j}$ is the Kronecker symbol and $(V, \xi)$ is a chart centered at $e$ ) and set $X=$ $\left(X_{1}, \ldots, X_{n}\right) ; X$ is a $\mathbf{k}$-basis of $\mathfrak{g}$. By $(6.11)$, for any $i \in\{1, \ldots, n\} X_{i}$ can be identified with the k-valued distribution $(-1)^{|\alpha|} D_{\xi}^{\alpha} \delta_{e}$ where $\alpha \in \mathbb{N}^{n}$ is the above multi-index; then $\mathfrak{g}$ is identified with the $\mathbf{k}$-vector space consisting of all $\mathbf{k}$-valued distributions of order 1 with support $\{e\}$. By $([22],(17.14 .2))$, for any $Y, Z \in \mathfrak{g}$,

$$
[Y, Z] \triangleq Y \star Z-Z \star Y \in \mathfrak{g}
$$

and the bilinear map $[\bullet, \bullet]: \mathfrak{g} \times \mathfrak{g} \rightarrow \mathfrak{g}$ is a Lie bracket; $\mathfrak{g}$, endowed with this bracket, is called the Lie algebra of $\mathbf{G}([22],(19.3 .3))$. An ideal of $\mathfrak{g}$ is a $\mathbf{k}$-vector space $\mathfrak{a} \subseteq \mathfrak{g}$ such that $[\mathfrak{g}, \mathfrak{a}] \subseteq \mathfrak{a}$. The Lie algebra $\mathfrak{g}$ is called simple if is noncommutative and if its only proper ideal is $\{0\}$, semisimple if its only abelian ideal is $\{0\}$, nilpotent if there exists a finite decreasing sequence of ideals $\left(\mathfrak{g}_{i}\right)_{0 \leq i \leq p}$ such that $\mathfrak{g}_{0}=\mathfrak{g}, \mathfrak{g}_{p}=\{0\}$ with $\left[\mathfrak{g}, \mathfrak{g}_{i}\right] \subseteq \mathfrak{g}_{i+1}(0 \leq i \leq p-1)$, and solvable if there exists a sequence

$$
\mathfrak{g}_{0}=\mathfrak{g} \supsetneqq \mathfrak{g}_{1} \supsetneqq \cdots \supsetneqq \mathfrak{g}_{n}=\{0\}
$$

of Lie subalgebras such that $\mathfrak{g}_{i}$ is a one-codimensional ideal in $\mathfrak{g}_{i-1}, 1 \leq i \leq n([8]$, Subsect. I.5.1, Prop. 2). All nilpotent Lie algebras are solvable.

Assuming that the Lie group $\mathbf{G}$ is connected, it is called semisimple (resp. nilpotent, solvable) if its Lie algebra is semisimple (resp. nilpotent, solvable).

6.2.4. The universal enveloping algebra and its centre. More generally, given any associative k-algebra $A$, one can endow $A$ with a k-Lie algebra structure by defining the Lie bracket $[a, b]=a b-b a(a, b \in A)$. A representation of $\mathfrak{g}$ is defined to be a $\mathbf{k}$-Lie algebra morphism from $\mathfrak{g}$ to such $A$. There exists an associative $\mathbf{k}$-algebra $\mathrm{U}_{\mathbf{k}}(\mathfrak{g})$ (denoted by $\mathrm{U}(\mathfrak{g})$ if $\mathbf{k}=\mathbb{R}$ and $\mathrm{U}_{\mathbb{C}}(\mathfrak{g})$ if $\mathbf{k}=\mathbb{C}$ ) together with a representation 
$\theta: \mathfrak{g} \rightarrow \mathrm{U}_{\mathbf{k}}(\mathfrak{g})$ with the following universal property: for any associative $\mathbf{k}$-algebra representation $\varphi: \mathfrak{g} \rightarrow A$, there exists a unique $\mathbf{k}$-algebra morphism $\psi: \mathrm{U}_{\mathbf{k}}(\mathfrak{g}) \rightarrow A$ such that $\psi \theta=\varphi$. Thus $\mathrm{U}_{\mathbf{k}}(\mathfrak{g})$ is unique up to isomorphism and is called the universal enveloping algebra of $\mathfrak{g}$. Consider the standard monomials

$$
\left\{X_{i_{1}} X_{i_{2}} \ldots X_{i_{n}}: i_{j} \in\{1, \ldots, n\}, \quad i_{1} \leq \ldots \leq i_{n}\right\}
$$

According to the Poincaré-Birkhoff-Witt theorem, these standard monomials form a $\mathbf{k}$-basis of $\mathrm{U}_{\mathbf{k}}(\mathfrak{g}) ; \mathrm{U}_{\mathbf{k}}(\mathfrak{g})$ is a skew polynomial ring and a noncommutative left and right Noetherian integral domain $([42], \S 1.7)$.

If $\mathfrak{g}$ is the Lie algebra of a Lie group $\mathbf{G}$, then $\mathrm{U}_{\mathbf{k}}(\mathfrak{g})$ is isomorphic to, and identified with, $\mathcal{T}_{e}^{\infty}(\mathbf{G})$. The dual $\mathrm{U}_{\mathbf{k}}^{*}(\mathfrak{g}) \triangleq \operatorname{Hom}_{\mathbf{k}}\left(\mathrm{U}_{\mathbf{k}}(\mathfrak{g}), \mathbf{k}\right)$ is isomorphic to the power series ring $\mathbf{k}[[x]]$ (where $x=\left(x_{i}\right)_{1 \leq i \leq n}$ is a sequence of $n$ commuting indeterminates) and, according to E. Borel's theorem ([65], Theorem 38.1), there exists a canonical epimorphism $\mathcal{E}(\mathbf{G}) \rightarrow \mathrm{U}_{\mathbf{k}}^{*}(\mathfrak{g}): f \mapsto \sum_{\alpha \in \mathbb{N}^{n}} \frac{1}{\alpha !}\left(X^{\alpha} f\right) x^{\alpha}$, called the Borel epimorphism.

Let $P: \mathcal{E}(\mathbf{G}) \rightarrow \mathcal{E}(\mathbf{G})$ be a differential operator ([22], Sect. XVII.13). Such an operator is called left- (resp. right-) invariant if for any $a \in \mathbf{G}$ and $\varphi \in \mathcal{E}(\mathbf{G})$,

$$
P \varphi=\gamma(a)\left(P \gamma\left(a^{-1}\right) \varphi\right) \quad\left(\text { resp. } P \varphi=\delta(a)\left(P \delta\left(a^{-1}\right) \varphi\right)\right) .
$$

For short, a differential operator is called invariant when it is left-invariant, and bi-invariant when it is both left- and right-invariant. The algebra of all invariant (resp. bi-invariant) differential operators is identified with $U_{\mathbf{k}}(\mathfrak{g})$ (resp. $\mathrm{Z}_{\mathbf{k}}(\mathfrak{g})$, i.e. the centre of $\mathrm{U}_{\mathbf{k}}(\mathfrak{g})$, which is a commutative domain) ([22], (19.3.1), (23.36.6)).

If $\mathfrak{g}$ is semisimple, then $\mathrm{Z}_{\mathbf{k}}(\mathfrak{g})$ is the polynomial ring $\mathbf{k}\left[y_{1}, \ldots, y_{l}\right]$ where the natural integer $l$ is the "rank" of $\mathfrak{g}([23], \S \S 1.9 .8,7.3 .8)$, thus $\mathbf{Z}_{\mathbf{k}}(\mathfrak{g})$ is Noetherian. The case of solvable Lie algebras is quite different since there exists a nilpotent Lie algebra $\mathfrak{g}$ of dimension 45 such that $\mathrm{Z}_{\mathbf{k}}(\mathfrak{g})$ is not Noetherian $([23], \S 4.9 .20)$.

\section{REFERENCES}

[1] W. Abramczuk, A Class of Surjective Convolution Operators, Pacific J. of Math., 110 (1984), pp. 1-7.

[2] C.A. Berenstein and M.A. Dostal, The Ritt theorem in several variables, Ark. Mat., 12 (1974), pp. 267-280.

[3] J.-E. BJÖRK, Rings of operators, North Holland Mathematics Library, 1979.

[4] N. Bourbaki, Algèbre, Chapitres 1 à 3, Hermann, Paris, 1970. (English translation: Algebra I, Springer, 1989.)

[5] N. Bourbaki, Algèbre commutative, Chapitres 1-3 et 4-7, Masson, 1985. (English translation: Commutative Algebra, Chapters 1-7, Springer, 1989.)

[6] N. Bourbaki, Topologie générale, Chapitres 1 à 4, Hermann, Paris, 1971. (English translation: General Topology, Chapters 1-4, Springer, 1989.)

[7] N. Bourbaki, Espaces vectoriels topologiques, Masson, Paris, 1981. (English translation: Topological Vector Spaces, Springer, 1987.)

[8] N. Bourbaki, Groupes et algèbres de Lie, Chapitres 1 et 2-3, Hermann, Paris, 1971-1972. (English translation: Lie groups and Lie algebras, Chapters 1-3, Springer, 1989.)

[9] H. Bourlès and U. OBerst, Fundamental principle for analytic linear systems of partial differential-difference equations with constant coefficients, submitted to Math. Contr., Sign., Syst.

[10] D. Bréthé and J.J. Loiseau, Stabilization of linear time-delay systems, JESA-RAIRO-APII, 6 (1997), pp. 1025-1047.

[11] F. Bruhat, Sur les représentations induites des groupes de Lie, Bull. Soc. Math. de France, 84 (1956), pp. 97-205.

[12] F. BRUHAT, Lectures on Lie groups and representations of locally compact groups, Tata Institute of Fundamental Research, Bombay, 1958. 
[13] F. BRuhat, Distributions sur un groupe localement compact et applications à l'étude des représentations des groupes p-adiques, Bull. Soc. Math. de France, 89 (1961), pp. 43-75.

[14] J. Byrnes and G. Ostheimer (edts), Computational Noncommutative Algebra and Applications, Kluwer Academic Publishers, Dordrecht, 2004.

[15] A. Cerezo, F. Rouvière, Résolubilité locale d'un opérateur différentiel invariant du premier ordre, Ann. Sc. ENS, Série 4, 21-30, 1971.

[16] G.S. Chirikjian and A. Kyatkin, An Operational Calculus for the Euclidean Motion Group with Applications in Robotics and Polymer Science, The Journal of Fourier Analysis and Applications, 6 (2000), 583-606.

[17] E. Chizak, A. Quadrat and D. Robertz, Effective algorithms for parametrizing linear control systems over Ore algebras, Applicable Algebra in Engineering, Communications and Computing, 16 (2005), pp. 319-376.

[18] P.M. Cohn, Free Rings and their Relations, 2nd ed, Academic Press, London, 1985.

[19] P.M. Cohn, Basic Algebra -Groups, Rings and Fields, Springer, London, 2003.

[20] P.M. Conn, Further Algebra and Applications, Springer, London, 2003.

[21] S.C. Coutinho, A Primer of Algebraic D-modules, London Math. Soc. Texts 33, Cambridge University Press, Cambridge, 1995.

[22] J. Dieudonné, Eléments d'analyse, vol. 1-7, Bordas, Paris, 1969-1978. (English translation: Treatise on analysis, vol. I-VII, Academic Press, 1969-1978.)

[23] J. Dixmier, Algèbres enveloppantes, Gauthier-Villars, Paris, 1974. (English translation: Enveloping Algebras, AMS Bookstore, 1996.)

[24] M. Duflo And D. Wigner, Sur la résolubilité des équations différentielles invariantes sur un groupe de Lie, Séminaire Equations aux dérivées partielles (Ecole Polytechnique), 9 (1977-1978), pp. 1-7.

[25] L. Ehrenpreis, Solutions of some problems of division III, American J. of Mathematics, 78 (1956), pp. 685-715.

[26] L. Ehrenpreis, Solutions of some problems of division IV, American J. of Mathematics, 82 (1960), pp. 148-170.

[27] L. EHRENPREIS, Fourier analysis in several complex variables, Wiley, 1970.

[28] S.J.L. Eijndhoven And L.C.G.J.M Habets, Equivalence of Convolution Systems in a Behavioral Framework, Math. Contr., Sign., Syst., 16 (2003), pp. 175-206.

[29] M. FLIESS, Some basic structural properties of generalized linear systems, Systems \& Control Letters, 15 (1990), pp. 391-396.

[30] M. Fliess, J. Levine, Ph. Martin and P. Rouchon, Flatness and defect of non-linear systems: introducing theory and examples, Int. J. Control, 61 (1995), pp. 1327-1361.

[31] H. Gluesing-Luerssen, A behavioral approach to delay-differential systems, SIAM J. Control Optim., 35 (1997), pp. 480-499.

[32] H. Gluesing-Luerssen, Linear Delay-Differential Systems with Commensurate Delays: An Algebraic Approach, Springer, 2002.

[33] H. Gluesing-Luerssen, P. Vettori and S. Zampieri, The algebraic structure of DD systems: a behavioral perspective, Kybernetika, 37 (2001), pp. 397-426.

[34] D.I. Gurevič, Counterexamples to a problem of L. Schwartz, Funct. Anal. Appl., 9 (1975), pp. $116-120$.

[35] L.C.G.J.M HABETS, System equivalence for AR-systems over rings -with an application to delay-differential systems, Math. Contr., Sign., Syst., 12 (1999), pp. 219-244.

[36] M. Hall, The Theory of Groups, The MacMillan Comp., 1959.

[37] L. Hörmander, The Analysis of Linear Partial Differential Operators II, Springer, 1983.

[38] J.-J. Kelleher AND B.-A. TAYlor, Closed ideals in locally convex algebras of analytic functions, J. Reine Angew. Math., 255 (1972), pp. 190-209.

[39] T.Y. LAM, Lectures on Modules and Rings, Springer, New York, 1999.

[40] T.Y. Lam, Serre's Problem on Projective Modules, Springer, 2006.

[41] S. MacLane, Categories for the Working Mathematician, 2nd ed., Springer, New York, 1998.

[42] J.C. McConnel And J.C. Robson, Noncommutative Noetherian Rings, corrected ed., American Mathematical Society, 2001.

[43] P. Maisonobe and C. SabBah (dir.), D-modules cohérents et holonomes, Hermann, Paris, 1993.

[44] B. Malgrange, Existence et approximation des solutions des équations aux dérivées partielles et des équations de convolution, Ann. Inst. Fourier, 6 (1956), pp. 271-355.

[45] B. Malgrange, Sur les systèmes différentiels à coefficients constants, Séminaire Jean Leray (Collège de France, Paris), 7 (1961-1962), pp. 1-13.

[46] B. Malgrange, Systèmes différentiels à coefficients constants, Séminaire Bourbaki, 246 (19621963), pp. 1-11.

[47] B. Mitchell, Theory of Categories, Academic Press, New York, 1965. 
[48] H. Mounier, Propriétés structurelles des systèmes linéaires à retards: aspects théoriques et pratiques, Thèse de doctorat de l'Université Paris Sud, Orsay, 24 octobre 1995.

[49] H. Mounier, Algebraic interpretations of the spectral controllability of a linear delay system, Forum Mathematicum, 10 (1998), pp. 39-58.

[50] U. Oberst, Multidimensional Constant Linear Systems, Acta Applicandae Mathematicae, 20 (1990), pp. 1-175.

[51] V.P. Palamodov, Linear Differential Operators with Constant Coefficients, Springer, 1970.

[52] F. Parreau and Y. Weit, Schwartz's Theorem on Mean Periodic Vector-Valued Functions, Bull. Soc. Math. France, 117 (1989), pp. 319-325.

[53] H. Pillai and S. Shankar, A behavioral approach to control of distributed systems, SIAM J. Control Optim., 37 (1998), pp. 388-408.

[54] J.W. Polderman and J.C. Willems, Introduction to Mathematical Systems Theory $-A$ Behavioral Approach, Springer, 1998.

[55] J.-F. Pommaret, Partial Differential Control Theory, vol. 1 \& 2, Kluwer Academic Publishers, Dordrecht, 2001.

[56] J.-F. Pommaret and A. Quadrat, Localization and parametrization of linear multidimensional control systems, Systems \& Control Letters, 37 (1999), pp. 247-260.

[57] N. Popescu, Abelian Categories with Applications to Rings and Modules, Academic Press, 1973.

[58] P. Rocha And J.C. Willems, Behavioral Controllability of Delay-Differential Systems, SIAM J. Control Optim., 35 (1997), pp. 254-264.

[59] P. Rocha And J. Wood, A new perspective on controllability properties for dynamical systems, Applied Mathematics and Computer Science, 7 (1997), pp. 869-879.

[60] W. Rump, Almost abelian categories, Cahiers de topologie et géométrie différentielle catégoriques, 42(3) (2001), pp. 163-225.

[61] H. Schaefer, Topological Vector Spaces, 3rd Printing Corrected, Springer, 1971.

[62] L. Schwartz, Théorie des distributions (3rd ed.), Hermann, Paris, 1966.

[63] S. Shankar, The Nullstellensatz for Systems of PDE, Advances in Applied Mathematics, 23 (1999), pp. 360-374.

[64] S. Shankar, Geometric Completeness of Distribution Spaces, Acta Applicandae Mathematicae, 77 (2003), pp. 163-180.

[65] F. Trèves, Topological Vector Spaces, Distributions and Kernels, Academic Press, 1967.

[66] P. Vettori And S. Zampieri, Module theoretic approach of convolutional systems, Linear Algebra Appl., 351-352 (2002), pp. 739-759.

[67] P. Vettori and S. Zampieri, Some Results on Systems Described by Convolution Equations, IEEE Trans. on Automat. Control, 46 (2001), pp. 793-797.

[68] J.C. Willems, From time series to linear systems - Part I: Finite-dimensional linear time invariant systems, Automatica, 22 (1986), pp. 675-694.

[69] J.C. Willems, Models for dynamics, Dynamics Reported, 2 (1989), pp. 171-269.

[70] J.C. Willems, Paradigms and Puzzles in the Theory of Dynamical Systems, IEEE Trans. on Automat. Control, 36 (1991), pp. 259-294.

[71] J. Wood, Modules and Behaviours in nD Systems Theory, Multidimensional Systems and Signal Processing, 11 (2000), pp. 11-48.

[72] J. Wood, Key problems in the extension of module-behaviour duality, Linear Algebra Appl., 351-352 (2002), pp. 761-798.

[73] J. Wood, E. Rogers And D.H. Owens, Controllable and autonomous nD linear systems, Multidimensional Systems Signal Process., 10 (1999), pp. 63-69.

[74] J. Wood And E. ZERz, Notes on the definition of behavioural controllability, Systems \& Control Letters, 37 (1999), pp. 31-37.

[75] B. YAZICI, Stochastic Deconvolution over Groups, IEEE Trans. on Information Theory, 50 (2004), pp. 494-510.

[76] E. ZERZ, Discrete multidimensional systems over $\mathbb{Z}_{n}$, Systems \& Control Letters, 56 (2007), pp. $702-708$ 